\title{
1 Imprecise probabilistic estimation of design floods with epistemic
}

\section{2 uncertainties}

3

4 Wei Qi ${ }^{\mathrm{a}, \mathrm{b}}$, Chi Zhang ${ }^{\mathrm{a}^{*}}$, Guangtao Fu ${ }^{\mathrm{b}}$ and Huicheng Zhou

5

$6 \quad{ }^{a}$ School of Hydraulic Engineering, Dalian University of Technology, Dalian 116024, China

$7{ }^{\mathrm{b}}$ Centre for Water Systems, College of Engineering, Mathematics and Physical Sciences,

8 University of Exeter, North Park Road, Harrison Building, Exeter EX4 4QF, UK

$9 \quad{ }^{*}$ Corresponding author: email: czhang@dlut.edu.cn; Tel: +86 411 84708517; Fax: +86 411

$10 \quad 84708517$

11

12 key points:

13 An imprecise probabilistic approach is developed for design flood estimation

14 A new robustness criterion for design flood selection is proposed Interactions among various sources of uncertainty affect the total cost considerably

17 Abstract: An imprecise probabilistic framework for design flood estimation is proposed on the basis of the Dempster-Shafer theory to handle different epistemic uncertainties from data, probability distribution functions and probability distribution parameters. These uncertainties are incorporated in cost-benefit analysis to generate the lower and upper bounds of the total cost for flood control, thus presenting improved information for decision making on design floods. Within the total cost bounds, a new robustness criterion is proposed to select a design flood that can tolerate higher levels of uncertainty. A variance decomposition approach is used to quantify individual and interactive impacts of the uncertainty sources on total cost. Results from three case studies, with 127-, 104- and 54-year flood data sets respectively, show that the 
imprecise probabilistic approach effectively combines aleatory and epistemic uncertainties from the various sources and provides upper and lower bounds of the total cost. Between the total cost and the robustness of design floods, a clear trade-off which is beyond the information that can be provided by the conventional minimum cost criterion is identified. The interactions among data, distributions and parameters have a much higher contribution than parameters to the estimate of the total cost. It is found that the contributions of the various uncertainty sources and their interactions vary with different flood magnitude, but remain roughly the same with different return periods. This study demonstrates that the proposed methodology can effectively incorporate epistemic uncertainties in cost-benefit analysis of design floods.

Keywords: Decision making; Dempster-Shafer theory; Frequency analysis; Hydraulic design; Imprecise probability; Uncertainty

\section{Introduction}

Estimation of design flood discharge related to a specific return period plays a crucial role in flood management: for example, the design of hydraulic structures. Conventionally, Flood Frequency Analysis (FFA) is used to estimate design floods, i.e., fitting Probability Distribution Functions (PDFs) to observed flood data and deriving a design flood discharge through the extrapolation of the upper distribution tail to specified low exceedance probabilities [Merz and Blöschl, 2008]. Recently, cost-benefit analysis has been incorporated into FFA to compare different design floods and obtain a cost effective design value [Tung and Mays, 1981; Bao et al., 1987; Ganoulis, 2003; Jonkman et al., 2004; Abrishamchi et al., 2005; Rossi et al., 2005; Su and Tung, 2013a; Su and Tung, 2013b; Botto et al., 2014]. It has been proven that the design flood value calculated using cost-benefit analysis with the assumption of liner damage 
and cost functions is equivalent to the flood value from the conventional FFA method [Botto et al., 2014].

A key issue in design flood estimation is to quantify and reduce the various uncertainties from different sources [Wood and Rodríguez-Iturbe, 1975a; Wood and Rodríguez-Iturbe, 1975b; Bodo and Unny, 1976; Stedinger et al., 1993; Tanaka and Takara, 2002; Pandey et al., 2004; Beguería, 2005; Merz and Thieken, 2005; Su and Tung, 2013b]. The inherent variability of flood events which is of aleatory uncertainty in nature is represented using a PDF. Significant uncertainties exist regarding the PDF derivation, such as the use of insufficient historical data, selection of PDFs and estimation of PDF parameters; most of these uncertainties are epistemic in nature and related to imprecise and incomplete knowledge about flood systems [Merz and Thieken, 2005; Fu et al., 2011; Su and Tung, 2013b].

62

Previous research has analysed the respective effects of epistemic uncertainties in data, PDF selection and distribution parameters on design flood estimation. It has been illustrated that a longer length of data could reduce the uncertainty in design flood estimation and project benefits [Su and Tung, 2013a; Su and Tung, 2013b; Botto et al., 2014]. However, the uncertainties related to the use of the Peak Over Threshold (POT) series selection have not been analysed in terms of cost-benefit. PDF selection has been widely recognised as a major uncertainty source for flood frequency analysis [Kidson and Richards, 2005; Calenda et al., 2009; Rahman et al., 2013]. Many PDFs, such as Generalized Extreme Value (GEV) and 3parameter Lognormal (LN3), have been used for comparison [Botto et al., 2014], but the overall uncertainty from these PDFs which cannot be rejected using statistical tests has not been quantified and compared to other uncertainty sources. Parameter uncertainty of PDFs arises from parameter estimation approaches or data used in estimation, and has been 
represented using normal distribution [Su and Tung, 2013b] or using other distributions derived from the Monte Carlo approach [Tung and Mays, 1981; Bao et al., 1987; Botto et al., 2014].

Consequently, there is lack of understanding of combined and interactive contributions of different uncertainty sources to design flood estimates and lack of understanding of overall benefits of design options.

The various uncertainties should be represented and handled in a more holistic and coherent framework which will allow for a more realistic design flood estimation considering multiple uncertainty sources. Most recently, efforts have been made to systematically represent and quantify multiple uncertainty sources in a chain of models, such as investigating climatic impacts on hydrological systems and water resources management [Steinschneider et al., 2012], investigating impacts of precipitation and hydrological model uncertainties on discharge simulation uncertainty [Qi et al., 2016a], and investigating influence of parameter uncertainties on algorithm performance [Qi et al., 2016b]. Uncertainties in emission scenarios, global circulation models, downscaling methods and hydrological models have been quantified and their respective contributions to the overall output uncertainty have been compared [Vrugt et al., 2005; Wilby and Harris, 2006; Kay et al., 2009; Prudhomme and Davies, 2009; Bosshard et al., 2013]. In those prior studies, Monte Carlo based probabilistic approaches or sensitivity analysis approaches have been used. This holistic framework allows for identification of predominant sources of uncertainty and provides a more complete understanding of uncertainties in the modelling chain. To the best of our knowledge, a holistic framework has not been developed for design flood estimation, which requires simultaneous handling of multiple aleatory and epistemic uncertainty sources. 

approach that can effectively handle various aleatory and epistemic uncertainties through costbenefit analysis. In this approach, an imprecise probabilistic approach, based on DempsterShafer theory [Dempster, 1967; Shafer, 1976], is used to combine the epistemic uncertainties of data, probability distributions and their parameters. As a result, the lower and upper bounds of cumulative probabilities of flood can be generated and incorporated in cost-benefit analysis. The lower and upper total cost, including construction cost and expected flood damage cost, is then estimated explicitly. To select a robust design flood within the range of lower and upper total cost, a new criterion is proposed and contrasted with the conventional minimum total cost criterion. The individual and interactive contributions of different uncertainty sources to the overall uncertainty in estimating the total cost are quantified using a variance-based sensitivity analysis approach. Three case studies, with 54-year, 104-year and 127-year flood data

111 respectively, were used to test the newly proposed IPDF approach. Similar to Botto et al. [2014],

112 flood series were assumed to be stationary, i.e., the probability of occurrence of an extreme 113 event in the current or any future year is the same [Olsen et al., 1998]. In each case study, three 114 probability distributions were selected on the basis of the Anderson-Darling (A-D) test and different data sets were generated using POT and Annual Maximum (AM) methods to represent epistemic uncertainties. In this paper, there are three advancements from the present state of

117 knowledge: (1) a new and holistic imprecise probabilistic estimation approach for design flood

118 estimation is proposed and demonstrated to integrate aleatory and epistemic uncertainties; (2)

119 a new robustness criterion is proposed and demonstrated to select a design flood that can 120 tolerate higher levels of uncertainty, and a clear trade-off between the total cost and the robustness of design floods is identified, which is beyond the information that can be provided

122 by previous research; and (3) the variance decomposition approach is used to quantify 123 individual and interactive impacts of uncertainty sources on total cost, and it is found that the 
124 interactions among data, distributions and parameters affect the total cost considerably. These

125 findings represent state-of-the-art knowledge in design flood estimation. The research of this

126 paper could be used to evaluate design options and guide efforts to reduce the uncertainty from

127 multiple epistemic uncertainties in design flood estimation.

128

129 This paper is divided into seven sections. Section 2 provides an overview of three case studies

130 and the related epistemic uncertainties in data selection, probability distribution fitting and

131 probability distribution parameters. Section 3 introduces the IPDF approach. The numerical

132 procedures to implement the IPDF approach are described in section 4. Applications of the

133 IPDF approach to real-world cases are presented in section 5. Discussion and conclusions are

134 presented in section 6 and section 7.

135

136 2. Case studies

137 Three case studies of different flood record lengths are used in this study. These are selected

138 from three rivers of different climates and different catchment areas: Yangtze River in south

139 China, Songhuajiang and Biliu rivers in northeast China. Yangtze River is the largest rive in

140 China with a catchment area of 2 million $\mathrm{km}^{2}$, and is dominated by a sub-tropical humid

141 monsoon climate with abundant rainfall. Songhuajiang is the third largest river in China with

142 a catchment area of 0.56 million $\mathrm{km}^{2}$, and is characterized by a temperate monsoon climate

143 with long winter, aridness and low temperature. Biliu is a medium scale basin with a catchment

144 area of $2814 \mathrm{~km}^{2}$, and is characterized by a temperate monsoon marine climate. The daily flow

145 records are from 1882 to 2008 (127 years) at the Three Gorges gauge station of Yangtze River,

146 from 1898 to 2001 (104 years) at the Harbin site of Songhuajiang, and from 1958 to 2011 (54

147 years) at the Biliu gauge of Biliu. 


\subsection{Data selection uncertainty}

150 Hydrological data are generally associated with different sources of uncertainties including

151 data quality, representative data period selection, AM or POT series selection and length of

152 time series, as summarised by Merz and Thieken [2005]. In this study, data uncertainty arises

153 from the selection of historical data, represented by different data sets generated using AM and

154 POT methods, and other data uncertainties are not considered.

156 Selection of a threshold value is normally based on expert judgement [Beguería, 2005], and its

157 impact on design flood estimation is not fully understood [Tanaka and Takara, 2002; Pandey et al., 2004; Beguería, 2005]. To analyse the data uncertainty, a series of threshold values were adopted to generate flood series of different sizes, as shown in Table 1 . The dependence between flood flows at different time steps was not considered, similar to other studies [Coles et al., 2003; Kidson and Richards, 2005; Calenda et al., 2009; Xu et al., 2009], as the impacts caused by ignoring the dependence seem negligible [Rosbjerg, 1985; Xu et al., 2009].

163

\subsection{Probability distribution uncertainty}

165 In FFA, the probability $\mathrm{P}\left[Q \geq q_{T}\right]$ of a $T$-year flood $q_{T}$ (the flood is exceeded once in $T$ years on average) can be defined as

$$
\mathrm{P}\left[Q \geq q_{T} \mid \boldsymbol{\theta}\right]=\int_{q_{T}}^{\infty} f(q \mid \boldsymbol{\theta}) d q
$$

168 where $Q$ denotes the random flow variable and $f(q \mid \theta)$ denotes a PDF corresponding to the

169 Cumulative Distribution Function (CDF) $F(q \mid \theta)$. With the AM series, the sampling interval

170 of observed floods is one year, so the number of events is automatically one per year. With the

171 POT series, the number of occurrences of events in a given year is a random variable. Assuming

172 a Poisson process [Cunnane, 1979; Onoz and Bayazit, 2001], the return period of $Q$, in years, 
173 can be calculated as [Rosbjerg, 1985; Rosbjerg et al., 1992; Madsen et al., 1997; Beguería, 174 2005; Bhunya et al., 2012; Bhunya et al., 2013]:

$$
T=\frac{1}{\lambda \cdot \mathrm{P}\left[Q \geq q_{T} \mid \boldsymbol{\theta}\right]}
$$

where $\lambda$ is the mean number of occurrences per year, and $T$ is the return period.

Many probability distributions have been proposed to simulate the true, unknown probability distribution of flood in the literature [Stedinger et al., 1993; Kidson and Richards, 2005]. There are three main approaches for distribution selection: official recommendation [Kidson and Richards, 2005], experience knowledge based selection [Merz and Thieken, 2005; Viglione et al., 2013] and statistical test based selection using methods such as L-Moments and A-D test

183 [Chowdhury et al., 1991; Di Baldassarre et al., 2009; Kjeldsen and Prosdocimi, 2015]. A single distribution is often recommended for use in an entire country due to simplicity and practicality, and this approach is used by many countries in the world, though there is no theoretical basis [Calenda et al., 2009]. The selection based on goodness-of-fit tests is not conclusive and this does not support the view that only one candidate distribution should be selected as there may be several distributions that pass statistical tests [Kidson and Richards, 2005; Calenda et al., 2009; Laio et al., 2009; Rahman et al., 2013]. In the sense that many candidate distributions cannot be rejected, each can be considered as a possible distribution. The uncertainty resulting from probability distribution selection is referred to as distribution uncertainty hereafter.

193 A-D test is normally used to assess the goodness-of-fit of different distributions and it is suggested that it has good performance for extreme events as it gives more weight to the tails than the Kolmogorov-Smirnov test [Palynchuk and Guo, 2008; Calenda et al., 2009; Haddad and Rahman, 2010]. The null hypothesis is that the data follow a specified distribution. This 
197 hypothesis is rejected at the chosen significance level if the test statistic, $A^{2}$, is greater than the 198 relevant critical value.

200 The A-D test results are shown in Table 2. Three distributions - GEV, Generalized Logistic

201 (GLO) and LN3 - are shown. The maximum likelihood method was used to estimate

202 distribution parameter values, and their probability density functions and cumulative 203 probability density functions are shown in Appendix A. It should be noted that critical values 204 of the A-D test vary with probability distribution types, distribution parameters and 205 significance levels. D'Agostino and Stephens [1986; Table 4.32] introduced two approaches to 206 calculate the critical values: empirical distribution function based approach [Stephens, 1974;

207 Stephens, 1976; Stephens, 1977; Stephens, 1979; Ahmad et al., 1988] and normalized spacing 208 based approach [Lockhart et al., 1986b]. It is argued that the latter is better than the former as

209 it does not depend on a specific parameter estimation method [Lockhart et al., 1986a]. 210 According to D'Agostino and Stephens [1986; Table 4.32], the critical values based on 211 normalized spacings for GEV, Logistic and Normal distributions are 3.00, 3.41 and 2.73 at a

212 significance level of 0.01 , respectively. These critical values are used as a reference, as other 213 statistics available for three-parameter distributions are not reliable [Laio, 2004]. All the 214 distributions in Table 2 pass the test.

\section{$216 \quad 2.3$ Parameter uncertainty}

217 After the selection of data sets and probability distributions, the parameter uncertainty will 218 arise in distribution parameter value estimation because of the limited length of the data sets. 219 For considering the parameter uncertainty, parameter uncertainty bounds were first defined. 220 Many methods are available to define parameter bounds of probability distributions: for example, subjective definition of an interval or perturbation around optimal estimates to 
generate lower and upper parameter bounds [Blazkova and Beven, 2002; Liang et al., 2011; Le

223 Coz et al., 2014]; subjective definition of parameter distribution with known parameters [Reis and Stedinger, 2005; Ribatet et al., 2006; Lee and Kim, 2008; Su and Tung, 2013b]; and using regional information to define mean and variance of parameters [Perreault et al., 2000]. In this paper, because no prior information on distribution parameters was known, the perturbation method was used. The percentage perturbation of parameters was derived through trial and error to ensure all the observed extreme flow data were bracketed by the resulting lower and upper flow bounds. In this study, the posterior probability is calculated according to the Generalized Likelihood Uncertainty Estimation (GLUE) approach [Beven and Binley, 1992; Beven and Freer, 2001], which is equivalent to the importance sampling approach [Nott et al., 2012]. The incorporation of the parameter uncertainty into total cost calculation will be presented in Eqs. (8), (11), (14) and (19) in Section 3.

\section{Imprecise probabilistic framework for design flood estimation}

The IPDF approach is illustrated in Fig. 1. This new approach includes five components. (a) The first is uncertainty characterisation of different sources, i.e., different probability distributions, their distribution parameters and different data thresholds (three thresholds $T_{1}, T_{i}$ and $\mathrm{T}_{\mathrm{n}}$ are shown for illustration). (b) The second is uncertainty combination using evidence theory, which results in lower and upper bounds of probabilities. (c) The third is cost-benefit analysis to show the variations of total cost. The uncertainty of the total cost is propagated from the imprecise probabilities of flow. (d) The fourth is sensitivity analysis to quantify individual and interactive contributions of different uncertainty sources using a variance decomposition method: the ANalysis Of VAriance (ANOVA). ANOVA can identify important uncertainty sources, and guide efforts to reduce uncertainty. (e) The fifth component is a new robustness 
$247\left(\left[D_{1}, D_{n}\right]\right)$. In this interval, different design floods can be selected and compared based on their

248 robustness which is evaluated by measuring the variations of total cost with different

249 uncertainty levels (as illustrated by $\mu_{0}, \mu_{\mathrm{i}}$ and $\mu_{\mathrm{n}}$ ). Steps (a) - (d) can be repeated to reduce the

250 uncertainty of total cost when new data or distribution models are included. Compared to

251 previous methods [e.g., Su and Tung, 2013a, b; Botto et al., 2014], this new IPDF approach

252 provides the upper and lower bounds of minimum total cost for a specific $T$-year design flood,

253 as a result of considering different types of epistemic uncertainties. Details of each component

254 are presented in the following subsections.

\subsection{Dempster-Shafer theory of evidence}

257 Dempster-Shafer theory of evidence is a kind of set-valued and evidence-based theory and can 258 describe overall uncertainties of stochastic and epistemic nature. It can handle uncertainties 259 from different aleatory and epistemic sources [Hall, 2003; Hall and Lawry, 2004; Hall et al., 260 2004; Fu et al., 2011]. This theory has been used in many fields, such as water distribution system design [Fu and Kapelan, 2011], evaluation of sewer flooding [Fu et al., 2011], groundwater flow and transport simulation [Ross et al., 2009], reliability analysis [Tonon et al., 2000], climate change [Hall et al., 2007], and rainfall-runoff modelling [Maskey et al., 2004].

264 One main difference from the Bayesian theory is that the Dempster-Shafer theory admits 265 imprecision in probability (e.g., a probabilistic interval), whilst the Bayesian theory assumes that uncertainty should always be measured by a single probability [Walley, 1991; Hall, 2003;

267 Fu and Kapelan, 2011]. From this point of view, the Dempster-Shafer theory can be regarded as a generalization of probability theory to cope with a problem for which information is not enough for an assignment of a single probability. Many uncertainties in design flood estimation are epistemic and do not allow the assignment of a single probability value due to insufficient 
information or conflicting evidence, thus it is promising to apply the evidence theory to handle various uncertainties.

273

274 In Dempster-Shafer theory the minimum and maximum amounts of evidence can be taken into 275 consideration to construct probability. For example, suppose that based on evidence $\Psi_{1}$, the 276 probability of a set of states $\Phi=\left\{\Phi_{1}, \cdots, \Phi_{n}\right\}$ which relate to interests $\Omega$ (e.g., the probability of 277 flood events) can be assigned as $\mathrm{P}\left[\Phi_{i} \mid \Psi_{1}\right]=p_{i}$, while using another evidence $\Psi_{2}$, the probability of a set of states $\Theta=\left\{\Theta_{1}, \cdots, \Theta_{k}\right\}$ which also relate to interests $\Omega$ can be assigned as $\mathrm{P}\left[\Theta_{j} \mid \Psi_{2}\right]=q_{j}$. The Dempster-Shafer theory of evidence can be used to combine $p_{i}$ with $q_{j}$ to give $m^{\Psi_{1}, \Psi_{2}}$ which represents beliefs assigned to interests $\Omega$ based on evidence $\Psi_{1}$ and $\Psi_{2}$.

Let $X$ be a universal nonempty set containing all the possible values of a variable $x$, and $P(x)$ is the power set of $X$, i.e., the set of all the subsets of $X$. Dempster-Shafer theory of evidence can be defined as a pair $(\xi, m)$, where $\xi$ is the family of nonempty element of $P(x)$ and $m$ is mapping

$$
m: \xi \rightarrow[0,1]
$$

287 Such that $m(\varnothing)=0$ and

$$
\sum_{A \in \xi} m(A)=1
$$

where $A \in P(X)$ and $m$ is called the basic probability assignment. The related imprecision of probability can be bounded at the lower end by a belief function

$$
\operatorname{Bel}(E)=\sum_{A \subset E} m(A)
$$

and at the upper end by a plausibility function

$$
P l(E)=\sum_{A \cap E \neq \varnothing} m(A)=1-\operatorname{Bel}(\bar{E})
$$


294 where $\bar{E}$ is the complement of $E$. The $\operatorname{Bel}(E)$ measures the minimum amount of evidence that 295 fully supports $x \in E \cdot P l(E)$ measures the maximum amount of evidence that could be linked 296 with the event $E$.

297

\section{$298 \quad 3.2$ Annual expected damage cost estimation}

299 For hydrologic structures, the Annual Expected Damage Cost (AEDC) can be defined below

300 using the probability $\mathrm{P}\left[Q \geq q_{T}\right]$ in Eq. (1)

$$
E\left(D \mid q_{T}^{*}, \boldsymbol{\theta}\right)=\int_{q_{T}^{*}}^{\infty} D(q) \cdot f(q \mid \boldsymbol{\theta}) d q
$$

where $q_{T}^{*}$ is the $T$-year flow capacity of hydraulic structures; $D(q)$ represents the flooddamage function corresponding to a flood magnitude of $q$ and $\boldsymbol{\theta}$ represents the parameters.

304 When parameter uncertainty is considered, the expected damage Eq. (7) can be written as [Bao et al., 1987]

$$
E\left(D \mid q_{T}^{*}, \boldsymbol{\theta}, S\right)=\int_{\mathcal{q}_{T}^{*}}^{\infty} D(q) \cdot \int_{\Theta} f(q \mid \boldsymbol{\theta}) \cdot \pi_{\boldsymbol{\theta} \mid S}(\boldsymbol{\theta} \mid S) d \boldsymbol{\theta} d q
$$

where $\Theta$ is the parameter space of a probability distribution; $S$ is the sample of flow data. The sampling distribution $\pi_{\theta \mid S}(\theta \mid S)$ can be calculated based on Bayesian theory as described below

$$
\pi_{\theta \mid S}(\theta \mid S)=\frac{\pi(\theta) \cdot l_{S \mid \theta}}{\int_{\Theta} \pi(\theta) \cdot l_{S \mid \theta}}
$$

311 where $\pi(\boldsymbol{\theta})$ is the prior probability of parameter $\boldsymbol{\theta} ; \pi_{\theta \mid S}(\boldsymbol{\theta} \mid S)$ is the posterior probability of

312 parameter $\boldsymbol{\theta} ; l_{S \mid \boldsymbol{\theta}}$ is likelihood function

$$
l_{S \mid \theta}=\prod_{i=1}^{N} f\left(x_{i} \mid \theta\right)
$$

314 where $N$ represents the total number of sampled flow data. 
316 Based on the total probability theorem the predictive distribution is obtained

$$
f^{P D}(q)=\int_{\Theta} f(q \mid \boldsymbol{\theta}) \cdot \pi_{\theta \mid S}(\boldsymbol{\theta} \mid S) d \boldsymbol{\theta}
$$

318 An analytical solution of Eq. (11) can be derived only for a few probability distributions 319 [Stedinger, 1983; Kuczera, 1999; Fawcett and Walshaw, 2015], and in practice, Monte Carlo 320 method can be used to calculate the integral in Eq. (11). When data uncertainty and distribution

321 uncertainty are considered, $f^{P D}(q)$ is not unique and the lower and upper bounds of imprecise

322 probabilities, $\underline{f^{P D}(q)}$ and $\overline{f^{P D}(q)}$, can be defined as

$$
\begin{aligned}
& \underline{f^{P D}(q)}=\inf _{e} f_{e}^{P D}(q) \\
& \overline{f^{P D}(q)}=\sup _{e} f_{e}^{P D}(q)
\end{aligned}
$$

where $e$ represents the $e$ th probability function. $f^{P D}(q)$ and $\overline{f^{P D}(q)}$ correspond to $\operatorname{Bel}(E)$ and $\operatorname{Pl}(E)$ in the sense of the Dempster-Shafer theory of evidence. The lower probability

$\underline{f^{P D}(q)}$ measures the minimum probability that evidence can fully support, i.e., the minimum 328 probability calculated from selected data and probability distributions, and the upper probability $\overline{f^{P D}(q)}$ measures the maximum probability that evidence can potentially support,

330 i.e., the maximum probability calculated from selected data and probability distributions. The 331 interval formed with Eqs. (12) and (13) provides a bracketing of a series of probabilities and its spread represents the extent of incomplete knowledge and imprecise information about the unknown but true distribution. Combining Eq. (8) with Eqs. (12) and (13), AEDC considering

334 epistemic uncertainties can be defined as follows

$$
\left\{\begin{array}{l}
\frac{E\left(D \mid q_{T}^{*}, S\right)}{\overline{E\left(D \mid q_{T}^{*}, S\right)}}=\int_{q_{T}^{*}}^{\infty} D(q) \cdot \underline{f_{q_{T}^{*}}^{*} D(q) \cdot \overline{f^{P D}(q)} d q} d q
\end{array}\right.
$$


336 where $E\left(D \mid q_{T}^{*}, S\right)$ and $\overline{E\left(D \mid q_{T}^{*}, S\right)}$ represent the minimum and maximum values of AEDC estimation.

\subsection{Imprecise probabilistic estimation of design floods}

340 Assuming that the construction cost and damage functions are linear and represented as $c \cdot q_{T}^{*}$

341 and $d \cdot\left(q-q_{T}^{*}\right)$, respectively, the total cost function is described below according to Eq. (8)

$$
C_{\text {total }}\left(q_{T}^{*} \mid \boldsymbol{\theta}, S\right)=c \cdot q_{T}^{*}+\int_{q_{T}^{*}}^{\infty} d \cdot\left(q-q_{T}^{*}\right) \cdot \int_{\Theta} f(q \mid \boldsymbol{\theta}) \cdot \pi_{\theta \mid S}(\boldsymbol{\theta} \mid S) d \boldsymbol{\theta} d q
$$

and can also be written as

$$
C_{\text {total }}\left(q_{T}^{*} \mid S\right)=c \cdot q_{T}^{*}+\int_{q_{T}^{*}}^{\infty} d \cdot\left(q-q_{T}^{*}\right) \cdot f^{P D}(q) d q
$$

where $c$ and $d$ are parameters.

There exists a deterministic relationship between parameters $c$ and $d$ and return period $T$ under the linear cost and damage assumption according to Botto et al. [2014]

$$
\frac{d}{c}=\frac{1}{\mathrm{P}\left[Q \geq q_{T}^{*} \mid S\right]}=T
$$

which is derived by minimizing Eq. (16) using AM data, i.e., taking the derivative of the total cost function with respect to $q_{T}^{*}$ and setting it to 0 . When POT data sets are considered, the above relationship becomes

$$
\frac{d}{c}=\frac{1}{\lambda \cdot \mathrm{P}\left[Q \geq q_{T}^{*} \mid S\right]}=T
$$

Eq. (18) is a generalization of Eq. (17), i.e., $\lambda$ equals to 1 for AM data set. In this paper, the flood-damage data are available for Biliu, and a linear function is fitted with $d=1.891$. For Three Gorges and Harbin case studies, the same value of $d$ is assumed since damage data are not available. 
359 When data uncertainty and distribution uncertainty are considered, the total cost function Eq.

$360 \quad$ (16) becomes

$$
\left\{\begin{array}{l}
\frac{C_{\text {total }}\left(q_{T}^{*} \mid S\right)}{\overline{C_{\text {total }}\left(q_{T}^{*} \mid S\right)}}=c \cdot q_{T}^{*}+\int_{q_{T}^{*}}^{\infty} \lambda(q) \cdot d \cdot\left(q-q_{T}^{*}\right) \cdot \underline{q_{T}^{*}+\int_{q_{T}^{*}}^{\infty} \lambda(q) \cdot d \cdot\left(q-q_{T}^{*}\right) \cdot \overline{f^{P D}(q)} d q} d q
\end{array}\right.
$$

which shows the lower and upper bounds of the total cost, incorporating epistemic uncertainties from data, probability distribution, and parameter uncertainties into the aleatory uncertainty of flood.

\subsection{Robustness criterion}

367 The minimum total cost criterion can be used to select the design flood. This criterion is employed in the case of one single total cost curve generated as in the study of Botto et al. [2014]. In the case of imprecise probabilities, a range of total costs can be obtained, bounded by the lower and upper curves. The total cost intervals provide an indication of the magnitude of total cost uncertainty which is faced by the decision maker when selecting a design flood, and with these intervals, the selection of a design flood depends on the preference of the decision maker or the use of decision criteria. However, the minimum total cost criterion [Botto et al., 2014] or the expected opportunity loss criterion [Su and Tung, 2013a; Su and Tung, 2013b] can be used for cases where parameter uncertainty is considered only.

A robustness criterion is proposed here to analyse the differences of design floods. The robustness, defined in the sense of the Info-gap theory [Ben-Haim, 2006; Hine and Hall, 2010], seeks a design value that can make a system maintain its prescribed functions over a range of uncertainty levels. In design flood estimation, robustness involves connecting $C_{\text {total }}$ with decision variation $q_{T}$ under an uncertainty level of $\partial$ : 


$$
\hat{\partial}\left(q_{T}, r_{c}\right)=\max \left(\partial: \min C_{\text {total }}\left(q_{T} \mid \partial\right) \geq r_{c}\right)
$$

383 where $r_{c}$ is a critical level of $C_{\text {total }}$. This critical level can be assumed to be the minimum

$384 C_{\text {total }}$ under an uncertainty level of $\partial$, thus robustness can be interpreted as the variation of the 385 minimum $C_{\text {total }}$ under many discrete uncertainty levels of $\partial$ [Matrosov et al., 2013]. The smaller the variations at different uncertainty levels, the more robust the design flood.

\subsection{Variance decomposition}

ANOVA is used to analyse the respective contributions of data, distributions, distribution parameters and their interactions to the overall uncertainty in total cost, $C_{\text {total }}$. Fig. 2 depicts the combinations employed in the uncertainty decomposition. To relate $C_{\text {total }}$ to the uncertainty sources, the superscripts $j, k$ and $l$ in $C_{\text {total }}^{j, k, l}$ are used to represent a combination of data set $j$, distribution $k$ and parameters $l$. Two cases, without parameter uncertainty (using the estimated optimal parameters) and with parameter uncertainty (using predictive probability distributions), are considered.

\subsubsection{Subsampling approach}

398 It has been argued that the ANOVA approach is based on a biased variance estimator that underestimates the variance when the sample size is small [Bosshard et al., 2013]. To reduce

400 the effect of the biased estimator on quantification of variance contribution, Bosshard et al. 401 [2013] proposed a subsampling method, which was used in this paper. In each subsampling iteration, $i$, we select two data sets out of all data sets analysed, and the superscript $j$ (data set)

403 in calculating $C_{\text {total }}^{j, k, l}$ is replaced with $\mathbf{g}(h, i)$. In the case of Three Gorges, the time series is 404 divided into nine non-overlapping subsets resulting in $9 ! /(2 !(9-2) !)=36$ possible combinations 405 of two elements, and correspondingly the superscript $\mathbf{g}$ is a $2 \times 36$ matrix as follows 


$$
\mathbf{g}=\left(\begin{array}{lllllllllllll}
1 & 1 & \cdots & 1 & 2 & 2 & \cdots & 6 & 6 & 6 & 7 & 7 & 8 \\
2 & 3 & \cdots & 9 & 3 & 4 & \cdots & 7 & 8 & 9 & 8 & 9 & 9
\end{array}\right)
$$

407 Similarly the superscript $\mathbf{g}$ is a $2 \times 28$ matrix in the case study of Harbin and a $2 \times 15$ matrix in 408 the case study of Biliu.

409

410

\subsubsection{ANOVA approach}

411 Based on the ANOVA method, the total sum of squares (SST) of $C_{\text {total }}$ can be divided into 412 sums of squares of the individual effects (with SSA, SSB and SSC corresponding to the 413 contribution of data, probability distributions and parameters respectively) and of their 414 interactions (SSI) as follows:

$$
\mathrm{SST}=\mathrm{SSA}+\mathrm{SSB}+\mathrm{SSC}+\mathrm{SSI}
$$

416

The terms can be estimated using the subsampling procedure as follows [Bosshard et al., 2013]:

$$
\begin{gathered}
\mathrm{SST}_{i}=\sum_{h=1}^{H} \sum_{k=1}^{K} \sum_{l=1}^{L}\left(C^{\mathbf{g}(h, i), k, l}-C^{\mathrm{g}(o, i), o, o}\right)^{2} \\
\mathrm{SSA}_{i}=K \cdot L \cdot \sum_{h=1}^{H}\left(C^{\mathbf{g}(h, i), o, o}-C^{\mathbf{g}(o, i), o, o}\right)^{2} \\
\mathrm{SSB}_{i}=H \cdot L \cdot \sum_{k=1}^{K}\left(C^{\mathbf{g}(o, i), k, o}-C^{\mathbf{g}(o, i), o, o}\right)^{2} \\
\mathrm{SSC}_{i}=H \cdot K \cdot \sum_{l=1}^{L}\left(C^{\mathbf{g}(o, i), o, l}-C^{\mathbf{g}(o, i), o, o}\right)^{2} \\
\operatorname{SSI}_{i}=\sum_{h=1}^{H} \sum_{k=1}^{K} \sum_{l=1}^{L}\left(C^{\mathbf{g}(h, i), k, l}-C^{\mathbf{g}(h, i), o, o}-C^{\mathbf{g}(o, i), k, o}-C^{\mathbf{g}(o, i), o, l}+2 \cdot C^{\mathbf{g}(o, i), o, o}\right)^{2}
\end{gathered}
$$

where symbol ${ }^{\circ}$ indicates averaging over the particular index. Then the contribution of each 424 uncertainty source $\eta^{2}$ is calculated as follows:

$$
\eta_{\mathrm{data}}^{2}=\frac{1}{I} \sum_{i=1}^{I} \frac{\mathrm{SSA}_{i}}{\mathrm{SST}_{i}}
$$




$$
\eta_{\text {distribution }}^{2}=\frac{1}{I} \sum_{i=1}^{I} \frac{\mathrm{SSB}_{i}}{\mathrm{SST}_{i}}
$$

$$
\eta_{\mathrm{parameter}}^{2}=\frac{1}{I} \sum_{i=1}^{I} \frac{\mathrm{SSC}_{i}}{\mathrm{SST}_{i}}
$$

$$
\eta_{\text {interaction }}^{2}=\frac{1}{I} \sum_{i=1}^{I} \frac{\mathrm{SSI}_{i}}{\mathrm{SST}_{i}}
$$

$429 \eta^{2}$ has a value between 0 and 1 , which represent $0 \%$ and $100 \%$ of contribution to the overall 430 uncertainty of the total cost respectively.

\section{Implementation of the proposed new approach}

433

434

435

436

437

This section is devoted to describe how the new proposed IPDF approach can be applied in practice. The numerical procedures are implemented according to the following main steps:

1. Uncertainties are clearly identified: e.g., data selection, probability distribution and distribution parameter uncertainty, which forms the basis of the implementation (see Fig. 1a). 2. The imprecise probabilities are quantified on the basis of Eqs. (12) and (13). The upper and lower probability bounds can be obtained (see Fig. 1b).

3. Once the uncertainties are quantified, Eq. (19) is applied to calculate total costs. Two total cost curves can then be obtained: lower and upper total cost bounds (see Fig. 1c).

4. The ANOVA approach is applied to quantify the contributions of uncertainties to the total cost uncertainty on the basis of Eqs. (21)-(31) (see Fig. 1d).

5. The robustness criterion is applied to evaluate flood value estimates based on Eq. (20) (see Fig. 1e). This criterion could be provided to decision maker for informed decision making.

It should be noted that, in the first step, although the identification of the uncertainties is subjective, this procedure enables a rigorous evaluation of the respective and combined impacts of the uncertainties and thus provides an enhanced understanding of their impacts on the 
449 selection of design floods. Its application to three real-world cases is described below in Section 4505.

\section{Application to real-world cases}

In this section, the newly proposed methodology is demonstrated step by step in the subsections from 5.1 to 5.4. Section 5.1 first shows imprecise probability characteristics of flood through integration of the uncertainties in data selection, probability distributions and parameters, derived from Eqs. (12) and (13). Section 5.2 shows the total cost derived from Eq. (19). Section

5.3 discusses the use of a new robustness criterion for design flood selection. Section 5.4 discusses the contributions of different uncertainty sources to the overall uncertainty in total costs using the variance decomposition method.

460

461

\subsection{Imprecise probability}

In this study, a Monte Carlo based method was used to compute the posterior distributions using GLUE. 2000 parameter sets for each distribution were sampled within the parameter uncertainty bounds using Latin Hypercube Sampling. Sampling (larger parameter sets were also used obtaining similar results) 2000 parameter sets were used in the research.

Fig. 3 shows the sampling distributions of a specific design flood obtained on the basis of the posterior parameter distributions of GEV, GLO, and LN3 in Biliu, Three Gorges and Harbin.

Note that the posterior distributions are reduced to a single curve when integrated via Eq. (11).

470 Cumulative probability curves in each panel represent different data sets under the same 471 probability distribution. In the case of Biliu, the curves span a large range, while most curves

472 from the other two case studies are closer to each other, except for one curve (i.e., AM in the 473 case of Three Gorges and T3 in the case of Harbin). The big departures of AM in Three Gorges 
and T3 in Harbin imply high uncertainties in flood estimation when the corresponding data sets

475

476

477

478

479

480

481

482

483

484

485

486

487

488

489

490

491

492

493

494

495

496

497

498 are considered only. Recall that the specific distributions cannot be rejected under each of the data sets using the A-D test. However, the spread of the distribution curves clearly shows the epistemic uncertainties in the selection of data sets. Similarly, comparing the differences in each panel reveals the significant epistemic uncertainties in the selection of distributions.

Fig. 4 shows the imprecise cumulative probability distributions of Biliu, Three Gorges and Harbin, respectively, when data and distribution uncertainties are incorporated with parameter uncertainty. For each individual probability distribution (GEV, GLO and LN3), as shown in the panels $(a-i)$ of the first three rows, the probability of each flood value is calculated based on predictive distributions and the intervals are derived from the selected data sets, i.e., 6 data sets, 9 data sets and 8 data sets for Biliu, Three Gorges and Harbin, respectively. The overall CDFs in the panels $(j-l)$ of the fourth row result from the selected data sets listed in Table 1 , probability distributions (GEV, GLO and LN3) and calculated predictive distributions related to parameter uncertainty using Eq.(11). In the case of Biliu, the overall probability bounds are roughly the same as those of each individual distribution, implying the distribution uncertainty has less impact than the data uncertainty. However, in the case of Three Gorges, the overall bounds are primarily determined by the bounds of $\mathrm{LN} 3$, implying that the distribution uncertainty is the dominating uncertainty source. The case of Harbin shows a mixed impact from both data and distribution uncertainties. This is compared with the study of Botto et al. [2014] where only one predictive distribution was generated when considering the uncertainty of distribution parameters only. Theoretically this predictive distribution should lie within the grey areas, i.e., bracketed by the lower and upper probabilistic bounds, because in this research data selection, probability distribution and parameter uncertainties all are considered and the bounds represent the minimum and maximum probabilities. 


\subsection{Imprecise probabilistic estimation of total cost}

501 Fig. 5 illustrates the lower and upper total cost bounds for the three case studies when data,

502 distribution and parameter uncertainties are considered. For any design flood value shown on $503 x$-axis, the lower and upper bounds of the total cost are represented by the two curves in each

504 panel. For each individual distribution in the panels $(a-i)$, the intervals illustrate the 505 uncertainties in data and distribution parameters; for the cases of overall uncertainty in the 506 panels $(j-l)$, the intervals illustrate the uncertainties in data, distributions and distribution 507 parameters. This is compared with the study of Botto et al. [2014] where only one curve was generated when considering the uncertainty of distribution parameters only.

510 In the case of Three Gorges, the differences of individual distributions in upper and lower total 511 cost bounds are remarkable, and in the cases of Biliu and Harbin, the differences are also 512 obvious, as shown in the first three rows of panels in Fig. 5. The overall upper and lower total 513 cost bounds are notably larger than those of each distribution in all the three cases, in particular, 514 in the cases of Biliu and Harbin. In the case of Three Gorges, the total cost bounds are mainly 515 affected by the uncertainty in the selection of distributions, while in the cases of Biliu and 516 Harbin, the influence of data sets and distributions on total cost bounds are all important. In 517 total cost calculation, the lower and upper probability bounds are multiplied by flood damage 518 and flood values, resulting in rather different total cost bounds due to their highly nonlinear relationships (e.g., as shown in Eq. (19)).

\subsection{Design flood selection using a robustness criterion}

In this study, 300 uncertainty levels were used. This means the uncertainty intervals from the

523 median CDF towards lower and upper bounds $\left(\overline{f^{P D}(q)}\right.$ and $\left.\underline{f^{P D}(q)}\right)$ in Fig. 4 were discretized 
into 300 subintervals. The variations of minimum total cost are thus calculated for each uncertainty level as shown in Fig. 6. The minimum total cost within each uncertainty level is shown on the $x$-axis, and robustness is shown on the $y$-axis under a set of uncertainty levels $(\alpha \%)$. Under each uncertainty level (except when $\alpha$ equals 1 ), the parameter $\lambda$ (recall Eq. (19)) was unknown, and the minimum value of $\lambda$ out of all selected data sets in each case study was used for calculating the minimum total cost. Two design flood selection criteria are compared in Fig. 6: the minimum total cost approach [Botto et al., 2014] and the robustness based approach. An $\alpha$ value of 0 means that the probability of a flood $q$ is determined by the median $\mathrm{CDF}$, while $\alpha=1$ represents the maximum deviation degree: upper and lower probability bounds. The minimum total cost curve corresponding to $\alpha=0$ is shown by the dashed lines in Fig. 5 .

The existence of robust decisions depends on both the degree of uncertainty and the richness of the available decision options [Lempert and Collins, 2007]. In this research, we did not try to find the robust decisions but to assess the robustness of options, thus the richness of options doesn't matter. The upper and lower total cost bounds correspond to different design floods with minimum total cost, and the optional design floods fall in an interval. To make an informed decision, the decision maker is presented with the intervals represented by the upper and lower total cost curves, though the exact design flood is unknown. Thus, for comparison with D1

542 which represents the results of the traditional minimum total cost criterion, D2 and D3 were

543 selected within the interval, representing two possible design floods that might be selected by 544 decision makers. It should be noted that D2 and D3 correspond to the minimum total cost of two total curves respectively. In Biliu, D1, D2 and D3 are $5700 \mathrm{~m}^{3} / \mathrm{s}, 8000 \mathrm{~m}^{3} / \mathrm{s}$ and 10,000

$546 \mathrm{~m}^{3} / \mathrm{s}$ respectively. In Three Gorges, they are 73,900 $\mathrm{m}^{3} / \mathrm{s}, 80,000 \mathrm{~m}^{3} / \mathrm{s}$ and $95,000 \mathrm{~m}^{3} / \mathrm{s}$ 547 respectively. In Harbin, they are $23,800 \mathrm{~m}^{3} / \mathrm{s}, 35,000 \mathrm{~m}^{3} / \mathrm{s}$ and $40,000 \mathrm{~m}^{3} / \mathrm{s}$ respectively. The 548 selected flood values are marked in Fig. 5. 
550 In Fig. 6, each curve represents a design flood, and its slope describes the variation of minimum

551 total cost with uncertainty $(\alpha \%)$. The steeper the slope is, the more robust the design is. If a 552 curve is on the right hand side of another, it has a larger minimum total cost. In the case of 553 Biliu, the curve of D1 is gentler than the other two designs, thus fewer changes in uncertainty 554 can result in larger perturbation in total cost. The robustness curves become steeper with an 555 increase in design floods from D1 to D3, thus the robustness increases, but the smallest minimum total cost increases as well, when $\alpha=1$. Similarly, in the cases of Three Gorges and Harbin, D1 options are less robust compared with D2 and D3, but the smallest minimum total cost of D2 and D3 is larger than D1. Between total cost and robustness there is a clear tradeoff which decision makers need to balance in the decision making process. Under some uncertainty levels, D1 has a larger minimum total cost than D2 or D3: for example, for $\alpha=0$ in

561 Biliu, D1 is larger than D2 but is smaller than D3, and in Three Gorges D1 is larger than D2 and D3, which results from the differences in the total cost curve corresponding to $\alpha=0$ (shown in Fig. 5 as dashed lines), and implies that smaller design floods do not mean smaller total cost and larger total cost does not mean robust designs. In Biliu and Harbin, the curves corresponding to $\alpha=0$ are close to the lower total cost bound, while in Three Gorges the result is different: the curves corresponding to $\alpha=0$ is close to the upper total cost bound. The differences may be because of the variations in upper and lower probability bounds and parameter $\lambda$ (recall Eq. (19)).

570 Although only three design flood values are selected for comparison, the results reveal the patterns: with an increasing design flood magnitude, more uncertainties can be tolerated while still guaranteeing the calculated total cost varies only slightly; thus the robustness increases, but the minimum total cost increases as well. Likewise, although 300 uncertainty levels were 
used, the results show the different robustness of design floods. Larger uncertainty level numbers were also analysed resulting similar robustness analysis results.

576

\section{$577 \quad 5.4$ Contributions of uncertainty sources}

578 Fig. 7 shows the breakdown results when applying ANOVA, i.e., the total cost curves of different data and probability distribution combinations under two cases: with parameter uncertainty (using predictive probability distributions - the first row panels) and without parameter uncertainty (using the estimated optimal parameters - the second row panels). It can be seen that total cost curves change with the variations of data set and probability distribution combinations. For example, in Biliu, there are $6 \times 3$ different total cost curves and these curves span large areas. Comparing the panels $(a-c)$ and $(d-f)$, it can be seen that the total cost curves are different in the two cases with and without parameter uncertainty. For example, in Three Gorges, when considering parameter uncertainty, the total cost curve of the AM-LN3 combination (the most upper total cost curve in Fig. 7b) moves up compared with the optimal parameter case (the most upper total cost curve in Fig. 7e).

590 As shown in Eqs. (16)-(19), the total cost is a function of return period $T$. Thus, the total cost 591 is different for different return period floods. On the basis of Eqs. (21)-(31), Fig. 8 shows the contributions of individual uncertainty sources, i.e., data selection, distribution and parameter uncertainties, and their interactions to the overall uncertainty in total cost in Biliu, Three

594 Gorges and Harbin for three return periods, i.e., 500-, 1000- and 2000-year, respectively. The 595 contributions of uncertainty sources are represented by the strips varying with flood values on 596 $x$-axis. 
598 In Biliu, regarding the 500 years return period, the contributions of data and distribution 599 uncertainty sources varies slightly with flood magnitude. Interactions which cannot be

600 considered in conventional FFA have a much higher contribution than parameter uncertainty,

601 and approximately have the same contribution as distributions. Other return periods in Biliu 602 show the same tendency. Similarly, in Three Gorges and Harbin, the contributions of 603 uncertainty sources vary significantly with flood magnitude but almost have no changes in 604 different return periods. The contribution of interactions is larger than parameter uncertainty in

605 Three Gorges and Harbin also. In Three Gorges and Harbin which have much longer flow 606 records than Biliu, with flow increases, contribution from interactions decreases and 607 contribution from distributions increases. Comparing the differences in the data contribution 608 among the three cases, the longer the data record, the less impact it has. For example, Three 609 Gorges with the longest data record (23-year longer than Harbin and 73-year longer than Biliu)

610 has the least impact from data uncertainty: the uncertainty contributions from data at most are 611 10.7\% in Three Gorges, 38.9\% in Harbin and 45.1\% in Biliu. The similar contributions of 612 different uncertainty sources in different return periods imply that the return periods have little 613 influence on the relative influences of different uncertainty sources.

614

\section{6. Discussion}

616 Botto et al. [2014] incorporated parameter uncertainty in the design flood estimation through

617 cost-benefit analysis, however, epistemic uncertainties from other sources, e.g., data and 618 probability distribution uncertainties, were not incorporated. Several studies have compared 619 the separate influence of data and distribution epistemic uncertainties in flood estimation 620 [Beguería, 2005; Merz and Thieken, 2005]. Bao et al. [1987] studied the influence of the number of data and four different probability distributions on annual expected damage cost separately. Su and Tung [2013b] studied the influence of different parameter estimation 
623 methods on flood damage. However, combining data, distribution and parameter uncertainties

624 in design flood estimation has not been investigated in the previous literature. The approach

625 proposed in this paper systematically combines the above mentioned aleatory and epistemic 626 uncertainties (data, probability distribution and distribution parameter uncertainties) in a 627 holistic framework.

628

629 Every single curve in Fig. 7 represents the results of total costs should the previous approach 630 proposed by Botto et al. [2014] be used. In the approach proposed by Botto et al. [2014], which 631 effectively addressed distribution parameter uncertainty in a cost-benefit analysis approach, 632 one single total cost curve is generated to find the optimal design flood estimate. Building on 633 this work, our approach can take other uncertainty sources (such as probability distribution and data selection uncertainties) into consideration, and thus generate uncertainty intervals (the 635 grey areas in Fig. 7). It can also be seen that the grey areas are different from the uncertainty 636 ranges spanned by all the total cost curves: for example, the lower bounds of the grey areas can 637 be larger (e.g., Figs. 7a and 7b) or smaller (e.g., Fig. 7c) than the ranges of all the total cost 638 curves. These differences are because the total cost calculation (Eq. (19)) considers data selection uncertainty, probability distribution uncertainty and parameter uncertainty and is very

640 different from previous approaches: for example, only considering parameter uncertainty 641 [Botto et al., 2014]. In addition, before a decision maker makes a decision, the design flood 642 value is a range using the newly developed approach, but the traditional approaches, such as 643 FFA and the approach proposed by Botto et al. [2014], provide decision maker a precise deign 644 flood value. The design flood values obtained from the newly proposed approach in this 645 research are shown to be no smaller than results using FFA and the UNcertainty COmpliant 646 DEsign (UNCODE) approach proposed by [Botto et al., 2014]. For example, Table 3 shows 647 the design flood estimates from the newly proposed IPDF approach, the UNCODE approach 
and FFA. In IPDF, the design flood intervals correspond to the minimum total costs in the lower and upper total cost bounds (to clearly show the minimum total costs in the lower total cost bounds, the minimum total costs in the upper total cost bounds are not shown in Fig. 7); in UNCODE, the design floods correspond to the minimum total costs among all the total cost curves shown in Fig. 7; in FFA, the design floods correspond to the minimum values among all the data set and distribution combinations. In the case of Three Gorges, the minimum 1000year flood from the newly proposed IPDF approach is $73,900 \mathrm{~m}^{3} / \mathrm{s}$, but it is $73,700 \mathrm{~m}^{3} / \mathrm{s}$ and $73,400 \mathrm{~m}^{3} / \mathrm{s}$ from UNCODE and FFA respectively (i.e., $0.3 \%$ and $0.7 \%$ smaller than the IPDF result respectively); in the cases of Harbin and Biliu, the minimum design floods of IPDF are no less than those from UNCODE and FFA. In addition, as shown in Table 3, IPDF provides design flood intervals which are not available in UNCODE and FFA. These intervals result from the considered uncertainties in data selection, probability distributions and distribution 660 parameters.

662 In the previous research, design flood selection was based on either return periods according 663 to flood frequency analysis or minimum total cost criterion according to cost-benefit analysis. 664 Compared with previous studies, in our research, a robustness criterion is introduced. This criterion can allow decision makers to analyse the sensitivity of calculated total cost to the variations of uncertainties. This information is particularly useful because it can be

667 incorporated in the decision making process to select the most robust design floods under deep uncertainties where data is scarce and distribution is unknown.

670 Sensitivity analysis can be conducted to explicitly evaluate the impact of uncertainty sources

671 on decision making [Van-Waveren et al., 2000; Xu and Tung, 2009], however, the newly 672 developed framework in our research can quantify the individual and interactive impacts of 
673 uncertainty sources in design flood estimation. As shown in this study, interactive influence 674 among different uncertainties can be significant (e.g., interactive contribution in Biliu is up to

$67545.1 \%$ ), and the importance of an uncertainty source can be underestimated without 676 considering its interactions with other sources. It should be noted that the uncertainty 677 contribution fractions obtained in this study are case-specific and might vary depending on the 678 specific uncertainty sources included. Further research on more case studies is required to 679 understand how the contribution fractions are affected by different uncertainty sources.

680

In addition to the epistemic uncertainties considered in this paper, other epistemic uncertainties can be explored using the new IPDF approach. For example, the number of distribution parameter sets used in the calculation of the sampling distributions and, as pointed out by Laio et al. [2009], the selection of significance level in PDF fitting. The higher the significance level, the more difficult the probability distributions can pass the test. In this study, with a significance level of 0.05 , GLO and LN3 for the T1 data and LN3 for the AM data in Three

687 Gorges could be rejected. Recall that these distributions cannot be rejected with a significance 688 level of 0.01 . The inclusion or exclusion of a specific distribution might have an impact on the lower and upper bounds of flood probabilities and thus on the ranges of total cost. However, this IPDF approach provides a quantitative means to measure the impacts and thus can better inform decision making.

692

693 It should be noted that the use of the GLUE approach to calculate the sampling distributions, 694 and the use of a trial and error approach to define the uncertainty bounds of the probability 695 distribution parameters are not necessary. Other approaches, such as importance sampling, 696 Metropolis-Hastings algorithm, Gibbs sampling, and the use of regional information to define 697 parameter bounds, can be applied as well. 


\section{Conclusions}

700 Accurate estimation of design flood plays a crucial role in flood management: for example, the design of hydraulic structures. However, the estimation is influenced by various uncertainties: for example, aleatory and epistemic uncertainties. The state-of-the-art methodologies in design flood estimation did not account for the aleatory and epistemic uncertainties simultaneously, evaluating the overall benefits of design flood options, and providing quantitative information about aleatory and epistemic uncertainty contributions and their interactive contributions to design flood estimation uncertainty. These have posed a long term challenge to hydrologists and engineers. This paper presents a state-of-the-art progress to meet the challenge. A holistic and coherent framework to allow for realistic design flood estimation under multiple uncertainties is developed. To illustrate the proposed methodology, three case studies with 127year, 104-year and 54-year flood data sets were employed. Three distributions were selected using the A-D test, and different data sets generated using AM and POT methods from historical flood data were considered. The major findings from this study are presented as

713 follows.

715 First, an imprecise probabilistic approach for design flood estimation is proposed. This approach effectively combines aleatory and epistemic uncertainties from data, probability distribution functions, and parameters on the basis of the Dempster-Shafer theory. It also presents upper and lower bounds of total cost faced by decision makers when selecting a design

719 flood.

Second, a robustness criterion for decision support in design flood selection is proposed. The design flood corresponding to the smallest minimum total cost can tolerate lower uncertainties, 
723 thus is not robust. With an increasing design flood magnitude, more uncertainties can be 724 tolerated while still guaranteeing the calculated total cost varies only slightly, thus the

725 robustness increases, but the minimum total cost increases as well. Between total cost and 726 robustness, there is a clear trade-off which decision makers need to balance in the decision 727 making process. This trade-off quantitatively provides the overall benefits of design flood 728 options, which provides an objective tool for decision makers to balance conflicting concerns.

730 Third, the interactions among data, distributions and parameters are significant and have a 731 much higher contribution than parameters to the uncertainty in total cost. The contributions of 732 data, distributions and parameters to the overall uncertaity in total cost vary with flood 733 magnitude. However, the contributions are almost the same for different return periods. This information implies that the overall uncertainty in estimated design floods could be underestimated if the interactions are disregarded, and therefore interactions should be considered in design flood estimation.

738 The approach proposed in this study could provide a blueprint for pragmatic flood frequency analysis under multiple epistemic uncertainties. Future research is encouraged to examine the applicability of the approach in other regions. In addition, climate change could influence flood

741 frequency analysis, and future research should focus on incorporating climate change impacts 742 into design flood estimation.

\section{Acknowledgements:}

745 This study was supported by the National Natural Science Foundation of China (Grant No. 74651320105010 and 51279021). The first author gratefully acknowledges the financial support 747 provided by the China Scholarship Council. The authors are deeply indebted to editors, Dr 
748 Francesco Serinaldi and another anonymous reviewer for their valuable time and constructive 749 suggestions that greatly improved the quality of this paper. The data of Three Gorges were 750 obtained from the China Three Gorges Corporation. The data of Biliu were obtained from the 751 Biliu reservoir administration. The data of Harbin were obtained from the Harbin hydrology 752 bureau. These data are available as in Supporting Information Data Set which includes Data

753 Set S1, Data Set S2 and Data Set S3. Data Set S1 corresponds to Three Gorges; Data Set S2 754 corresponds to Biliu; Data Set S3 corresponds to Harbin.

755

756

Appendix A: Probability density functions $(f)$ and cumulative probability 757 density functions $(F)$

758

The probability density functions $(f)$ and cumulative probability density functions $(F)$ used 759 in this paper are given in Eqs. (A1)-(A3):

$760 \quad$ Generalized Extreme Value distribution

761

$$
F(x)=\left\{\begin{array}{l}
\exp \left(-(1+k z)^{-1 / k}\right), k \neq 0 \\
\exp (-\exp (-z)), k=0
\end{array}, z \equiv \frac{x-\mu}{\sigma}\right.
$$

where $k, \sigma>0$ and $\mu$ are shape, scale and location parameter, respectively.

Generalized Logistic distribution

764

$$
F(x)=\left\{\begin{array}{l}
\frac{1}{1+(1+k z)^{-1 / k}}, k \neq 0 \\
\frac{1}{1+\exp (-z)}, k=0
\end{array}, z \equiv \frac{x-\mu}{\sigma}\right.
$$
where $k, \sigma>0$ and $\mu$ are shape, scale and location parameter, respectively.

$$
f(x)=\frac{\exp \left(-\frac{1}{2}\left(\frac{\ln (x-\gamma)-\mu}{\sigma}\right)^{2}\right)}{x \sigma \sqrt{2 \pi}}
$$


where $\mu, \sigma$ and $\gamma$ are, shape, scale and location parameter, respectively.

\section{References}

771 Abrishamchi, A., Ebrahimian, A., Tajrishi, M., Mariño, M., (2005). Case Study: Application of Multicriteria Decision Making to Urban Water Supply. Journal of Water Resources Planning and Management, 131(4): 326-335.

DOI:10.1061/(ASCE)07339496(2005)131:4(326)

775

Ahmad, M.I., Sinclair, C.D., Spurr, B.D., (1988). Assessment of flood frequency models using empirical distribution function statistics. Water Resources Research, 24(8): 1323-1328. DOI:10.1029/WR024i008p01323

Bao, Y., Tung, Y.-K., Hasfurther, V.R., (1987). Evaluation of uncertainty in flood magnitude estimator on annual expected damage costs of hydraulic structures. Water Resources Research, 23(11): 2023-2029. DOI:10.1029/WR023i011p02023

781

782

783

784

785

786

787

788

789

790

791

Beguería, S., (2005). Uncertainties in partial duration series modelling of extremes related to the choice of the threshold value. Journal of Hydrology, 303(1-4): 215-230. DOI:10.1016/j.jhydrol.2004.07.015

Ben-Haim, Y., 2006. Info-Gap Decision Theory: Decisions Under Severe Uncertainty, 2nd ed., Academic, London.

Beven, K., Binley, A., (1992). The future of distributed models: Model calibration and uncertainty prediction. Hydrological Processes, 6(3): 279-298. DOI:10.1002/hyp.3360060305

Beven, K.J., Freer, J.E., (2001). Equifinality, data assimilation, and uncertainty estimation in mechanistic modelling of complex environmental systems using the GLUE methodology. Journal of Hydrology, 249(1-4): 11-29. DOI:10.1016/S0022-1694(01)00421-8 
792 Bhunya, P.K., Berndtsson, R., Jain, S.K., Kumar, R., (2013). Flood analysis using negative binomial and Generalized Pareto models in partial duration series (PDS). Journal of Hydrology, 497: 121-132. DOI:10.1016/j.jhydrol.2013.05.047

795

796

797

798

799

800

801

802

803

804

805

806

807

808

809

810

811

812 Chowdhury, J.U., Stedinger, J.R., Lu, L.H., (1991). Goodness-of-fit tests for regional 813

814

815

816

Bhunya, P.K., Singh, R.D., Berndtsson, R., Panda, S.N., (2012). Flood analysis using generalized logistic models in partial duration series. Journal of Hydrology, 420-421: 5971. DOI:10.1016/j.jhydrol.2011.11.037

Blazkova, S., Beven, K., (2002). Flood frequency estimation by continuous simulation for a catchment treated as ungauged (with uncertainty). Water Resources Research, 38(8): 14-114-14. DOI:10.1029/2001wr000500

Bodo, B., Unny, T.E., (1976). Model uncertainty in flood frequency analysis and frequencybased design. Water Resources Research, 12(6): 1109-1117. DOI:10.1029/WR012i006p01109

Bosshard, T., Carambia, M., Goergen, K., Kotlarski, S., Krahe, P., Zappa, M., Schär, C., (2013). Quantifying uncertainty sources in an ensemble of hydrological climate-impact projections. Water Resources Research, 49(3): 1523-1536. DOI:10.1029/2011wr011533

Botto, A., Ganora, D., Laio, F., Claps, P., (2014). Uncertainty compliant design flood estimation. Water Resources Research, 50(5): 4242-4253. DOI:10.1002/2013wr014981

Calenda, G., Mancini, C.P., Volpi, E., (2009). Selection of the probabilistic model of extreme floods: The case of the River Tiber in Rome. Journal of Hydrology, 371(1-4): 1-11. DOI:10.1016/j.jhydrol.2009.03.010 generalized extreme value flood distributions. Water Resources Research, 27(7): 1765-1776.

\section{DOI:10.1029/91wr00077}

Coles, S., Pericchi, L.R., Sisson, S., (2003). A fully probabilistic approach to extreme rainfall modeling. Journal of Hydrology, 273(1-4): 35-50. DOI:10.1016/s0022-1694(02)00353-0 
817 Cunnane, C., (1979). A note on the Poisson assumption in partial duration series models. Water 818 Resources Research, 15(2): 489-494. DOI:10.1029/WR015i002p00489

819 D'Agostino, R.B., Stephens, M.A., 1986; Table 4.32. Goodness-of-Fit-Techniques. Taylor \& $820 \quad$ Francis.

821 Dempster, A.P., (1967). Upper and Lower Probabilities Induced by a Multivalued Mapping. 822 The Annals of Mathematical Statistics, 38(2): 325-339. DOI:10.2307/2239146

823 Di Baldassarre, G., Laio, F., Montanari, A., (2009). Design flood estimation using model 824 selection criteria. Physics and Chemistry of the Earth, 34(10-12): 606-611. 825 DOI:10.1016/j.pce.2008.10.066

826 Fawcett, L., Walshaw, D., (2015). Sea-surge and wind speed extremes: optimal estimation 827 strategies for planners and engineers. Stochastic Environmental Research and Risk 828 Assessment. DOI:10.1007/s00477-015-1132-3

829 Fu, G., Butler, D., Khu, S.-T., Sun, S.A., (2011). Imprecise probabilistic evaluation of sewer 830 flooding in urban drainage systems using random set theory. Water Resources Research, 831 47(2): W02534. DOI:10.1029/2009wr008944

832 Fu, G., Kapelan, Z., (2011). Fuzzy probabilistic design of water distribution networks. Water 833 Resources Research, 47(5). DOI:10.1029/2010wr009739

834 Ganoulis, J., (2003). Risk - based floodplain management: A case study from Greece. 835 International Journal of River Basin Management, 1(1): 41-47. DOI: $10.1080 / 15715124.2003 .9635191$

837 Haddad, K., Rahman, A., (2010). Selection of the best fit flood frequency distribution and 838 parameter estimation procedure: a case study for Tasmania in Australia. Stochastic 839 Environmental Research and Risk Assessment, 25(3): 415-428. DOI:10.1007/s00477-010840 $0412-1$ 
841 Hall, J., Fu, G., Lawry, J., (2007). Imprecise probabilities of climate change: aggregation of

842 fuzzy scenarios and model uncertainties. Climatic Change, 81(3-4): 265-281.

843 DOI: $10.1007 / \mathrm{s} 10584-006-9175-6$

844 Hall, J.W., (2003). Handling uncertainty in the hydroinformatic process. Journal of $845 \quad$ Hydroinformatics, 5(4): 215-232.

846 Hall, J.W., Lawry, J., (2004). Generation, combination and extension of random set 847 approximations to coherent lower and upper probabilities. Reliability Engineering \& System 848 Safety, 85(1-3): 89-101. DOI:10.1016/j.ress.2004.03.005

849 Hall, J.W., Rubio, E., Anderson, M.G., (2004). Random sets of probability measures in slope $850 \quad$ hydrology and stability analysis. Zeitschrift Angewandte Mathematik und Mechanik, 84(1011): 710-720. DOI:10.1002/zamm.200410146

852 Hine, D., Hall, J.W., (2010). Information gap analysis of flood model uncertainties and regional 853 frequency analysis. Water Resources Research, 46(1). DOI:10.1029/2008wr007620

854 Jonkman, S.N., Brinkhuis-Jak, M., Kok, M., (2004). Cost-benefit analysis and flood damage 855 mitigation in the Netherlands. Heron, 49: 95-111.

856 Kay, A.L., Davies, H.N., Bell, V.A., Jones, R.G., (2009). Comparison of uncertainty sources 857 for climate change impacts: flood frequency in England. Climatic Change, 92(1-2): 41-63. DOI:10.1007/s10584-008-9471-4

859 Kidson, R., Richards, K.S., (2005). Flood frequency analysis: assumptions and alternatives.

$860 \quad$ Progress in Physical Geography, 29(3): 392-410. DOI:10.1191/0309133305pp454ra

861 Kjeldsen, T.R., Prosdocimi, I., (2015). A bivariate extension of the Hosking and Wallis 862 goodness-of-fit measure for regional distributions. Water Resources Research, 51(2): 896907. DOI:10.1002/2014WR015912 
864 Kuczera, G., (1999). Comprehensive at-site flood frequency analysis using Monte Carlo

865 Bayesian inference. Water Resources Research, 35(5): 1551-1557.

866 DOI: $10.1029 / 1999 w r 900012$

867 Laio, F., (2004). Cramer-von Mises and Anderson-Darling goodness of fit tests for extreme

868 value distributions with unknown parameters. Water Resources Research, 40(9): W09308.

869 DOI:10.1029/2004wr003204

870 Laio, F., Di Baldassarre, G., Montanari, A., (2009). Model selection techniques for the 871 frequency analysis of hydrological extremes. Water Resources Research, 45(7). $872 \quad$ DOI: $10.1029 / 2007 w r 006666$

873 Le Coz, J., Renard, B., Bonnifait, L., Branger, F., Le Boursicaud, R., (2014). Combining 874 hydraulic knowledge and uncertain gaugings in the estimation of hydrometric rating curves: 875 A Bayesian approach. Journal of Hydrology, 509: 573-587. DOI:10.1016/j.jhydrol.2013.11.016

877 Lee, K.S., Kim, S.U., (2008). Identification of uncertainty in low flow frequency analysis using

878 Bayesian MCMC method. Hydrological Processes, 22(12): 1949-1964. 879 DOI: $10.1002 /$ hyp.6778

880 Lempert, R.J., Collins, M.T., (2007). Managing the risk of uncertain threshold responses: 881 comparison of robust, optimum, and precautionary approaches. Risk Analysis, 27(4): 1009882 26. DOI:10.1111/j.1539-6924.2007.00940.x

883 Liang, Z., Chang, W., Li, B., (2011). Bayesian flood frequency analysis in the light of model 884 and parameter uncertainties. Stochastic Environmental Research and Risk Assessment, 885 26(5): 721-730. DOI:10.1007/s00477-011-0552-y

886 Lockhart, R.A., O'Reilly, F.J., Stephens, M.A., (1986a). Test for the extreme value and weibull 887 distributions based on normalized spacings. Naval Research Logistics Quarterly, 33(3): 413-421. DOI:10.1002/nav.3800330307 
889 Lockhart, R.A., O'Reilly, F.J., Stephens, M.A., (1986b). Tests of Fit Based on Normalized $890 \quad$ Spacings. Journal of the Royal Statistical Society. Series B (Methodological), 48(3): 344$891 \quad 352$. DOI: $10.2307 / 2345431$

892 Madsen, H., Rasmussen, P.F., Rosbjerg, D., (1997). Comparison of annual maximum series 893 and partial duration series methods for modeling extreme hydrologic events: 1. At-site 894 modeling. Water Resources Research, 33(4): 747-757. DOI:10.1029/96WR03848

895 Maskey, S., Guinot, V., Price, R.K., (2004). Treatment of precipitation uncertainty in rainfall896 runoff modelling: a fuzzy set approach. Advances in Water Resources, 27(9): 889-898. 897 DOI:10.1016/j.advwatres.2004.07.001

898 Matrosov, E.S., Woods, A.M., Harou, J.J., (2013). Robust Decision Making and Info-Gap 899 Decision Theory for water resource system planning. Journal of Hydrology, 494: 43-58. $900 \quad$ DOI:10.1016/j.jhydrol.2013.03.006

901 Merz, B., Thieken, A.H., (2005). Separating natural and epistemic uncertainty in flood 902 frequency analysis. Journal of Hydrology, 309(1-4): 114-132. $903 \quad$ DOI:10.1016/j.jhydrol.2004.11.015

904 Merz, R., Blöschl, G., (2008). Flood frequency hydrology: 1. Temporal, spatial, and causal 905 expansion of information. Water Resources Research, 44(8). DOI:10.1029/2007WR006744 906 Nott, D.J., Marshall, L., Brown, J., (2012). Generalized likelihood uncertainty estimation 907 (GLUE) and approximate Bayesian computation: What's the connection? Water Resources $908 \quad$ Research, 48(12): W12602. DOI:10.1029/2011wr011128

909 Olsen, J.R., Lambert, J.H., Haimes, Y.Y., (1998). Risk of Extreme Events Under Nonstationary 910 Conditions. Risk Analysis, 18(4): 497-510. DOI:10.1111/j.1539-6924.1998.tb00364.x

911 Onoz, B., Bayazit, M., (2001). Effect of the occurrence process of the peaks over threshold on 912 the flood estimates. Journal of Hydrology, 244(1-2): 86-96. DOI:10.1016/s0022913 1694(01)00330-4 
914 Palynchuk, B., Guo, Y., (2008). Threshold analysis of rainstorm depth and duration statistics

915 at Toronto, Canada. Journal of Hydrology, 348(3-4): 535-545.

916 DOI:10.1016/j.jhydrol.2007.10.023

917 Pandey, M.D., Van Gelder, P.H.A.J.M., Vrijling, J.K., (2004). Dutch case studies of the

918 estimation of extreme quantiles and associated uncertainty by bootstrap simulations.

919 Environmetrics, 15(7): 687-699. DOI:10.1002/env.656

920 Perreault, L., Bernier, J., Bobée, B., Parent, E., (2000). Bayesian change-point analysis in 921 hydrometeorological time series. Part 1. The normal model revisited. Journal of Hydrology, 922 235(3-4): 221-241. DOI:10.1016/S0022-1694(00)00270-5

923 Prudhomme, C., Davies, H., (2009). Assessing uncertainties in climate change impact analyses 924 on the river flow regimes in the UK. Part 2: future climate. Climatic Change, 93(1-2): 197925 222. DOI:10.1007/s10584-008-9461-6

926 Qi, W., Zhang, C., Fu, G., Sweetapple, C., Zhou, H., (2016a). Evaluation of global fine927 resolution precipitation products and their uncertainty quantification in ensemble discharge 928 simulations. Hydrology and Earth System Sciences, 20(2): 903-920. DOI:10.5194/hess-20$929 \quad 903-2016$

930 Qi, W., Zhang, C., Fu, G., Zhou, H., (2016b). Quantifying dynamic sensitivity of optimization 931 algorithm parameters to improve hydrological model calibration. Journal of Hydrology, 533: 213-223. DOI:10.1016/j.jhydrol.2015.11.052

933 Rahman, A.S., Rahman, A., Zaman, M.A., Haddad, K., Ahsan, A., Imteaz, M., (2013). A study 934 on selection of probability distributions for at-site flood frequency analysis in Australia. 935 Natural Hazards, 69(3): 1803-1813. DOI:10.1007/s11069-013-0775-y

936 Reis, D.S., Stedinger, J.R., (2005). Bayesian MCMC flood frequency analysis with historical 937 information. Journal of Hydrology, 313(1-2): 97-116. DOI:10.1016/j.jhydrol.2005.02.028 
938 Ribatet, M., Sauquet, E., Grésillon, J.-M., Ouarda, T.B.M.J., (2006). A regional Bayesian POT 939 model for flood frequency analysis. Stochastic Environmental Research and Risk $940 \quad$ Assessment, 21(4): 327-339. DOI:10.1007/s00477-006-0068-z

941 Rosbjerg, D., (1985). Estimation in partial duration series with independent and dependent 942 peak values. Journal of Hydrology, 76(1-2): 183-195. DOI:10.1016/0022-1694(85)90098$943 \quad 8$

944 Rosbjerg, D., Madsen, H., Rasmussen, P.F., (1992). Prediction in partial duration series with 945 generalized pareto-distributed exceedances. Water Resources Research, 28(11): 3001-3010. 946 DOI:10.1029/92WR01750

947 Ross, J.L., Ozbek, M.M., Pinder, G.F., (2009). Aleatoric and epistemic uncertainty in 948 groundwater flow and transport simulation. Water Resources Research, 45(12): W00B15. 949 DOI:10.1029/2007wr006799

950 Rossi, G., Cancelliere, A., Giuliano, G., (2005). Case Study: Multicriteria Assessment of 951 Drought Mitigation Measures. Journal of Water Resources Planning and Management, 952

Shafer, G., 1976. A Mathematical Theory of Evidence. Princeton University Press, Princeton, 954 N. J.

Stedinger, J.R., (1983). Design events with specified flood risk. Water Resources Research, 956 19(2): 511-522. DOI:10.1029/WR019i002p00511

957 Stedinger, J.R., Vogel, R.M., Foufoula-Georgiou, E., 1993. Frequency analysis of extreme 958 events. Handbook of Hydrology. McGraw-Hill, New York.

959 Steinschneider, S., Polebitski, A., Brown, C., Letcher, B.H., (2012). Toward a statistical 960 framework to quantify the uncertainties of hydrologic response under climate change. Water 961 Resources Research, 48(11): W11525. DOI:10.1029/2011wr011318 
962 Stephens, M.A., (1974). EDF Statistics for Goodness of Fit and Some Comparisons. Journal 963 of the American Statistical Association, 69(347): 730-737. 964 DOI: $10.1080 / 01621459.1974 .10480196$

965 Stephens, M.A., (1976). Asymptotic Results for Goodness-of-Fit Statistics with Unknown 966 Parameters. 357-369. DOI:10.1214/aos/1176343411

967 Stephens, M.A., (1977). Goodness of Fit for the Extreme Value Distribution. Biometrika, 64(3): 968 583-588. DOI:10.2307/2345336

969 Stephens, M.A., (1979). Tests of Fit for the Logistic Distribution Based on the Empirical 970 Distribution Function. Biometrika, 66(3): 591-595. DOI:10.2307/2335180

971 Su, H.-T., Tung, Y.-K., (2013a). Flood-Damage-Reduction Project Evaluation with Explicit 972 Consideration of Damage Cost Uncertainty. Journal of Water Resources Planning and 973 Management, 139(6): 704-711. DOI:10.1061/(asce)wr.1943-5452.0000291

974 Su, H.-T., Tung, Y.-K., (2013b). Incorporating uncertainty of distribution parameters due to 975 sampling errors in flood-damage-reduction project evaluation. Water Resources Research, 976 49(3): 1680-1692. DOI:10.1002/wrcr.20116

977 Tanaka, S., Takara, K., 2002. A study on threshold selection in POT analysis of extreme floods, 978 Extremes of the Extremes: Extraordinary Floods. IAHS Publication. Int Assoc Hydrological 979 Sciences, Wallingford, pp. 299-304.

980 Tonon, F., Bernardini, A., Mammino, A., (2000). Reliability analysis of rock mass response by 981 means of Random Set Theory. Reliability Engineering \& System Safety, 70(3): 263-282. $982 \quad$ DOI:10.1016/S0951-8320(00)00059-4

983 Tung, Y.-K., Mays, L.W., (1981). Optimal risk-based design of flood levee systems. Water 984 Resources Research, 17(4): 843-852. DOI:10.1029/WR017i004p00843

985 Van-Waveren, R.H., Groot, S., Scholten, H., Geer, F.C.v., Wosten, J.H.M., Koeze, R.D., Noort, 986 J.J., 2000. Good Modelling Practice Handbook. STOWA, Utecht, The Netherland. 
987 Viglione, A., Merz, R., Salinas, J.L., Blöschl, G., (2013). Flood frequency hydrology: 3. A 988 Bayesian analysis. Water Resources Research, 49(2): 675-692. $989 \quad$ DOI: $10.1029 / 2011 w r 010782$

990 Vrugt, J.A., Diks, C.G.H., Gupta, H.V., Bouten, W., Verstraten, J.M., (2005). Improved 991 treatment of uncertainty in hydrologic modeling: Combining the strengths of global 992 optimization and data assimilation. Water Resources Research, 41(1): W01017. $993 \quad$ DOI: $10.1029 / 2004 w r 003059$

994 Walley, P., 1991. Statistical Reasoning with Imprecise Probabilities. Taylor \& Francis.

995 Wilby, R.L., Harris, I., (2006). A framework for assessing uncertainties in climate change 996 impacts: Low-flow scenarios for the River Thames, UK. Water Resources Research, 42(2): 997 W02419. DOI:10.1029/2005wr004065

998 Wood, E.F., Rodríguez-Iturbe, I., (1975a). A Bayesian approach to analyzing uncertainty 999 among flood frequency models. Water Resources Research, 11(6): 839-843. $1000 \quad$ DOI:10.1029/WR011i006p00839

1001 Wood, E.F., Rodríguez-Iturbe, I., (1975b). Bayesian inference and decision making for extreme 1002 hydrologic events. Water Resources Research, 11(4): 533-542. $1003 \quad$ DOI:10.1029/WR011i004p00533

1004 Xu, Y.-P., Booij, M.J., Tong, Y.-B., (2009). Uncertainty analysis in statistical modeling of 1005 extreme hydrological events. Stochastic Environmental Research and Risk Assessment, 1006 24(5): 567-578. DOI:10.1007/s00477-009-0337-8

1007 Xu, Y., Tung, Y., (2009). Decision Rules for Water Resources Management under Uncertainty. 1008 Journal of Water Resources Planning and Management, 135(3): 149-159. 1009 DOI:doi:10.1061/(ASCE)0733-9496(2009)135:3(149)

1010 
1011 Table 1 Flood discharge series generated using different thresholds and Annual Maximum

1012

(AM) approach from flood records in three cases

\begin{tabular}{|c|c|c|c|c|c|c|c|c|}
\hline \multicolumn{3}{|c|}{ Three Gorges } & \multicolumn{3}{|c|}{ Harbin } & \multicolumn{3}{|c|}{ Biliu } \\
\hline Symbol & $\begin{array}{c}\text { Threshold } \\
\text { level } \\
\left(\mathrm{m}^{3} / \mathrm{s}\right)\end{array}$ & $\begin{array}{l}\text { Number } \\
\text { of data }\end{array}$ & Symbol & $\begin{array}{l}\text { Threshold } \\
\text { level } \\
\left(\mathrm{m}^{3} / \mathrm{s}\right)\end{array}$ & $\begin{array}{l}\text { Number } \\
\text { of data }\end{array}$ & Symbol & $\begin{array}{c}\text { Threshold } \\
\text { level } \\
\left(\mathrm{m}^{3} / \mathrm{s}\right)\end{array}$ & $\begin{array}{l}\text { Number } \\
\text { of data }\end{array}$ \\
\hline $\mathrm{T} 1$ & 52,000 & 270 & $\mathrm{~T} 1$ & 6500 & 264 & $\mathrm{~T} 1$ & 500 & 105 \\
\hline $\mathrm{T} 2$ & 53,000 & 229 & $\mathrm{~T} 2$ & 7000 & 227 & $\mathrm{~T} 2$ & 700 & 62 \\
\hline $\mathrm{T} 3$ & 54,000 & 190 & $\mathrm{~T} 3$ & 7800 & 178 & $\mathrm{~T} 3$ & 737 & 54 \\
\hline $\mathrm{T} 4$ & 55,000 & 169 & $\mathrm{~T} 4$ & 8500 & 124 & $\mathrm{~T} 4$ & 1100 & 19 \\
\hline T5 & 56,000 & 135 & T5 & 9000 & 104 & T5 & 1300 & 13 \\
\hline $\mathrm{T} 6$ & 56,300 & 127 & T6 & 9500 & 88 & - & - & - \\
\hline $\mathrm{T} 7$ & 57,000 & 109 & $\mathrm{~T} 7$ & 10,000 & 77 & - & - & - \\
\hline $\mathrm{T} 8$ & 58,000 & 85 & - & - & - & - & - & - \\
\hline $\mathrm{AM}$ & - & 127 & $\mathrm{AM}$ & - & 104 & $\mathrm{AM}$ & - & 54 \\
\hline
\end{tabular}

1013

1014 
1015 Table 2 Anderson-Darling test results of three probability distributions in three cases

\begin{tabular}{|c|c|c|c|c|c|c|c|c|c|}
\hline & \multicolumn{3}{|c|}{ Three Gorges } & \multicolumn{3}{|c|}{ Harbin } & \multicolumn{3}{|c|}{ Biliu } \\
\hline & GEV & GLO & LN3 & GEV & GLO & LN3 & GEV & GLO & LN3 \\
\hline $\mathrm{T} 1$ & 1.57 & 2.46 & 2.02 & 1.29 & 1.97 & 1.39 & 0.34 & 0.41 & 0.47 \\
\hline $\mathrm{T} 2$ & 0.98 & 1.56 & 1.46 & 1.49 & 2.18 & 1.33 & 0.73 & 0.87 & 0.23 \\
\hline $\mathrm{T} 3$ & 0.62 & 1.14 & 0.56 & 0.90 & 1.07 & 0.59 & 0.60 & 0.73 & 0.33 \\
\hline $\mathrm{T} 4$ & 0.91 & 1.36 & 1.21 & 1.05 & 0.95 & 1.29 & 0.24 & 0.27 & 0.29 \\
\hline $\mathrm{T} 5$ & 0.54 & 0.91 & 0.45 & 0.89 & 0.77 & 1.06 & 0.18 & 0.20 & 0.18 \\
\hline T6 & 0.63 & 1.00 & 0.49 & 0.66 & 0.70 & 0.93 & - & - & - \\
\hline $\mathrm{T} 7$ & 0.67 & 0.99 & 0.51 & 0.90 & 1.07 & 0.59 & - & - & - \\
\hline $\mathrm{T} 8$ & 0.47 & 0.68 & 0.46 & - & - & - & - & - & - \\
\hline AM & 0.30 & 0.77 & 2.23 & 0.32 & 0.44 & 0.35 & 0.25 & 0.25 & 0.38 \\
\hline
\end{tabular}

1016 Note: GEV represents Generalized Extreme Value distribution; GLO represents Generalized

1017 Logistic distribution; LN3 represents 3-parameter Log-Normal distribution. AM represents

1018 annual maximum approach; the symbols from T1 to T8 represent different thresholds. 
1021 Table 3 Design flood estimates from the newly proposed Imprecise Probabilistic Design Flood 1022 (IPDF) approach, the UNcertainty COmpliant DEsign (UNCODE) approach proposed by 1023 [Botto et al., 2014] and Flood Frequency Analysis (FFA).

\begin{tabular}{cccc}
\hline & $\operatorname{IPDF}\left(\mathrm{m}^{3} / \mathrm{s}\right)$ & $\mathrm{UNCODE}\left(\mathrm{m}^{3} / \mathrm{s}\right)$ & $\mathrm{FFA}\left(\mathrm{m}^{3} / \mathrm{s}\right)$ \\
\cline { 2 - 4 } Three Gorges & {$[73,900 ; 113,200]$} & 73,700 & 73,400 \\
Harbin & {$[23,800 ; 63,800]$} & 23,800 & 22,800 \\
Biliu & {$[5700 ; 17,300]$} & 5700 & 5700
\end{tabular}

1024 Note: in IPDF, the design flood intervals correspond to the minimum total costs in the lower

1025 and upper total cost bounds; in UNCODE, the design floods correspond to the minimum total

1026 costs among all the total cost curves shows in Fig. 7; in FFA, the design floods correspond to 1027 the minimum values among all the data set and distribution combinations.

1028 


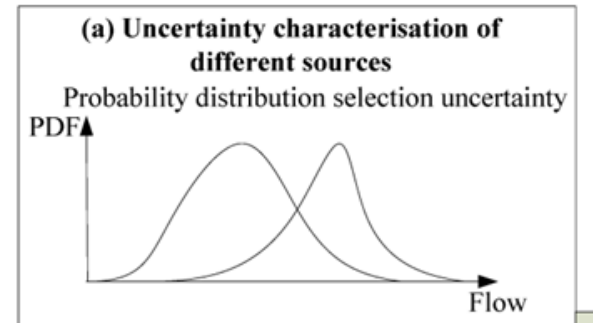

Probability distribution parameter uncertainty PDF\

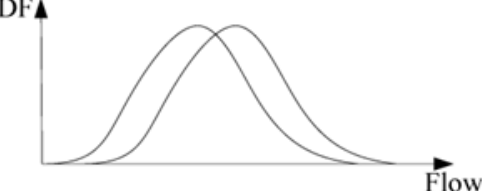

Data uncertainty from threshold selection
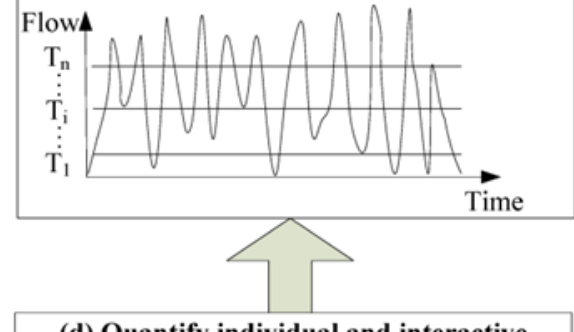
(d) Quantify individual and interactive contributions of different uncertainty sources using ANOVA

- Data $=$ Distribution $=$ Parameter Interaction

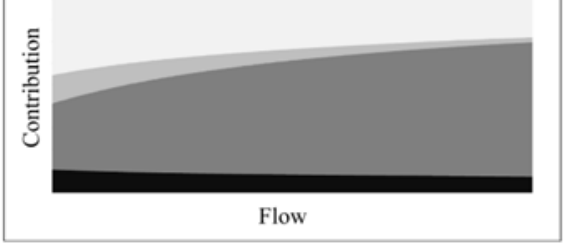

(b) Uncertainty combination using evidence theory: lower and upper bounds of probabilities

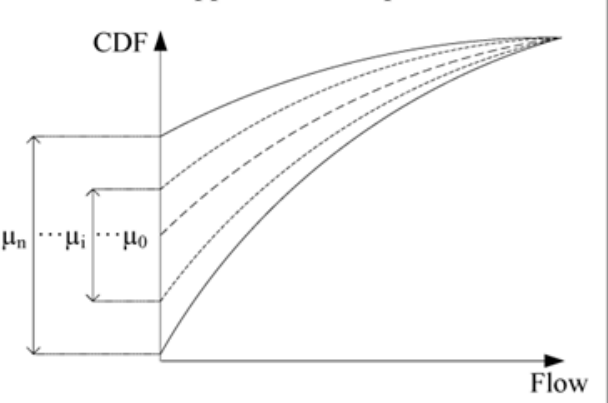

(c) Cost-benefit analysis using the newly proposed imprecise probabilistic estimation

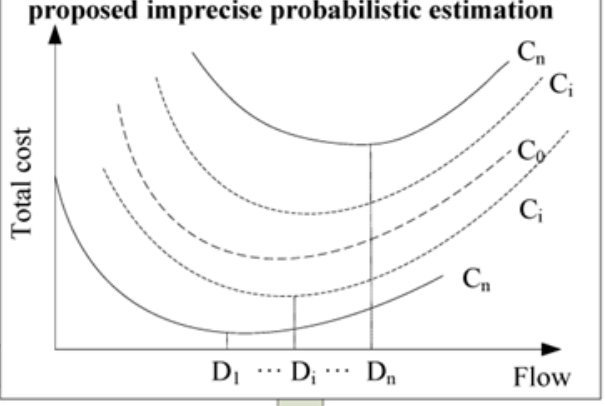

(e) Use of a new robustness criterion to select design flood

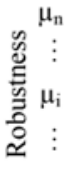

$D_{n}$

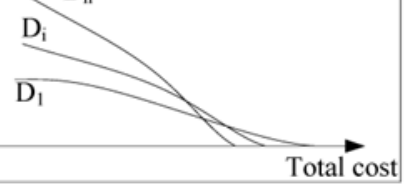

1031 Fig. 1 Diagrammatic representation of the proposed imprecise probabilistic framework for

1032 design flood estimation with epistemic uncertainties. Three thresholds $T_{1}, T_{i}$ and $T_{n}$ are shown

1033 for illustration; ANOVA represents the analysis of variance approach; $\mu_{0}, \mu_{\mathrm{i}}$ and $\mu_{\mathrm{n}}$ represent

1034 three different uncertainty levels; PDF represents Probability Distribution Functions; CDF

1035 represents Cumulative Distribution Function; $D_{1}, D_{i}$ and $D_{n}$ represent three different flood

1036 values; $\mathrm{C}_{1}, \mathrm{C}_{\mathrm{i}}$ and $\mathrm{C}_{\mathrm{n}}$ represent three different total cost values. 
1038

1039

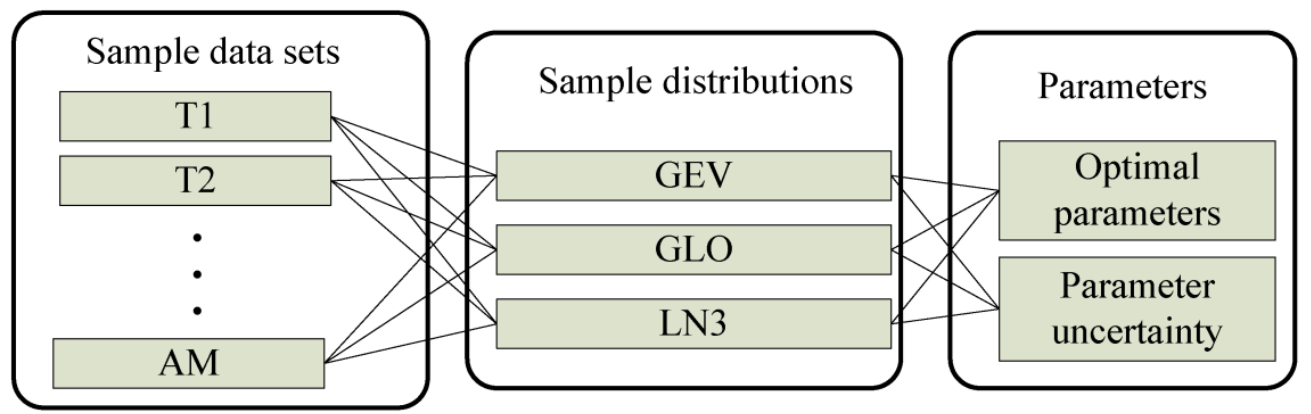

1040 Fig. 2 The combinations of data sets, distributions and parameters. T1, T2 and AM represent

1041 three selected data sets.

1042 

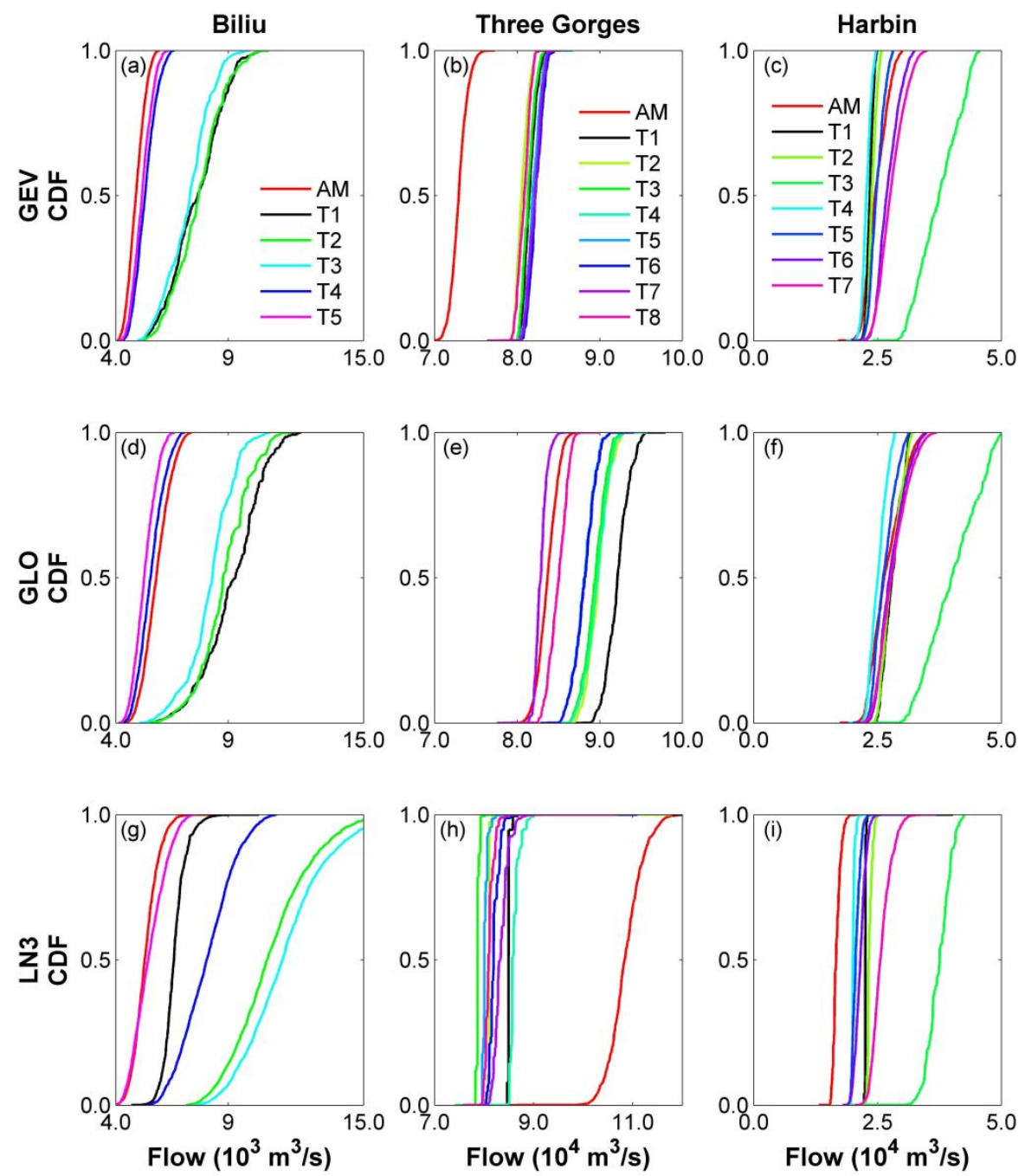

1043 Fig. 3 Sampling distributions of a specific design flood obtained using the posterior parameter

1045 distributions of GEV, GLO and LN3 in Biliu (a, d and g), Three Gorges (b, e and h) and Harbin 1046 (c, f and i). 

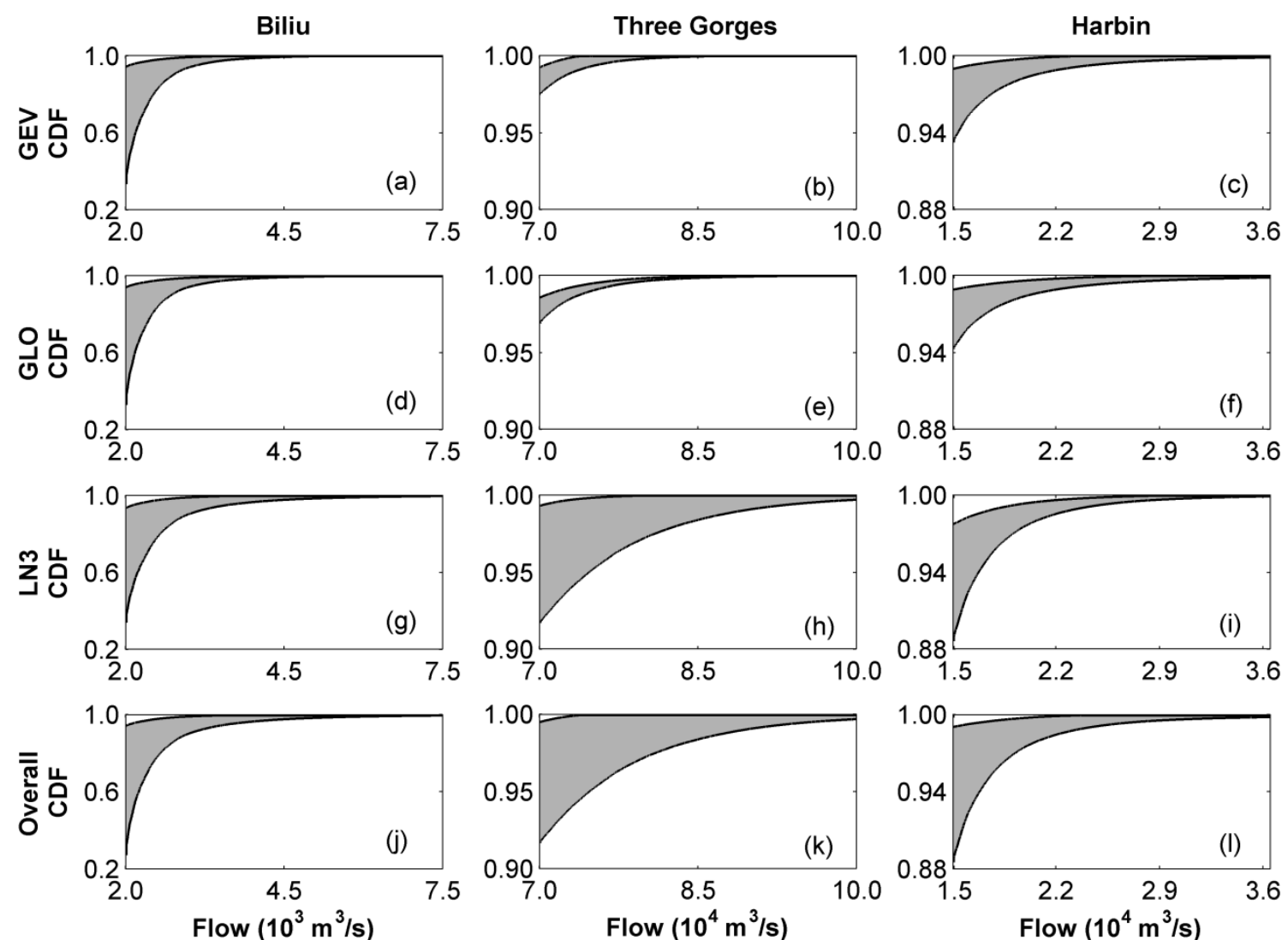

1048

Fig. 4 Lower and upper bounds of cumulative probabilities of flood for GEV, GLO, LN3 and

1050 combined distributions in Biliu (a, d, g and j), Three Gorges (b, e, h and k) and Harbin (c, f, i

1051 and 1) respectively. For each individual probability distribution (GEV, GLO and LN3), the

1052 probability of each flood value is calculated based on predictive distributions and the intervals

1053 are derived from the selected data sets. The combined CDFs ( $\mathrm{j}, \mathrm{k}$ and $\mathrm{l})$ result from selected

1054 data sets, probability distributions and calculated predictive distributions related to parameter

1055 uncertainty. 

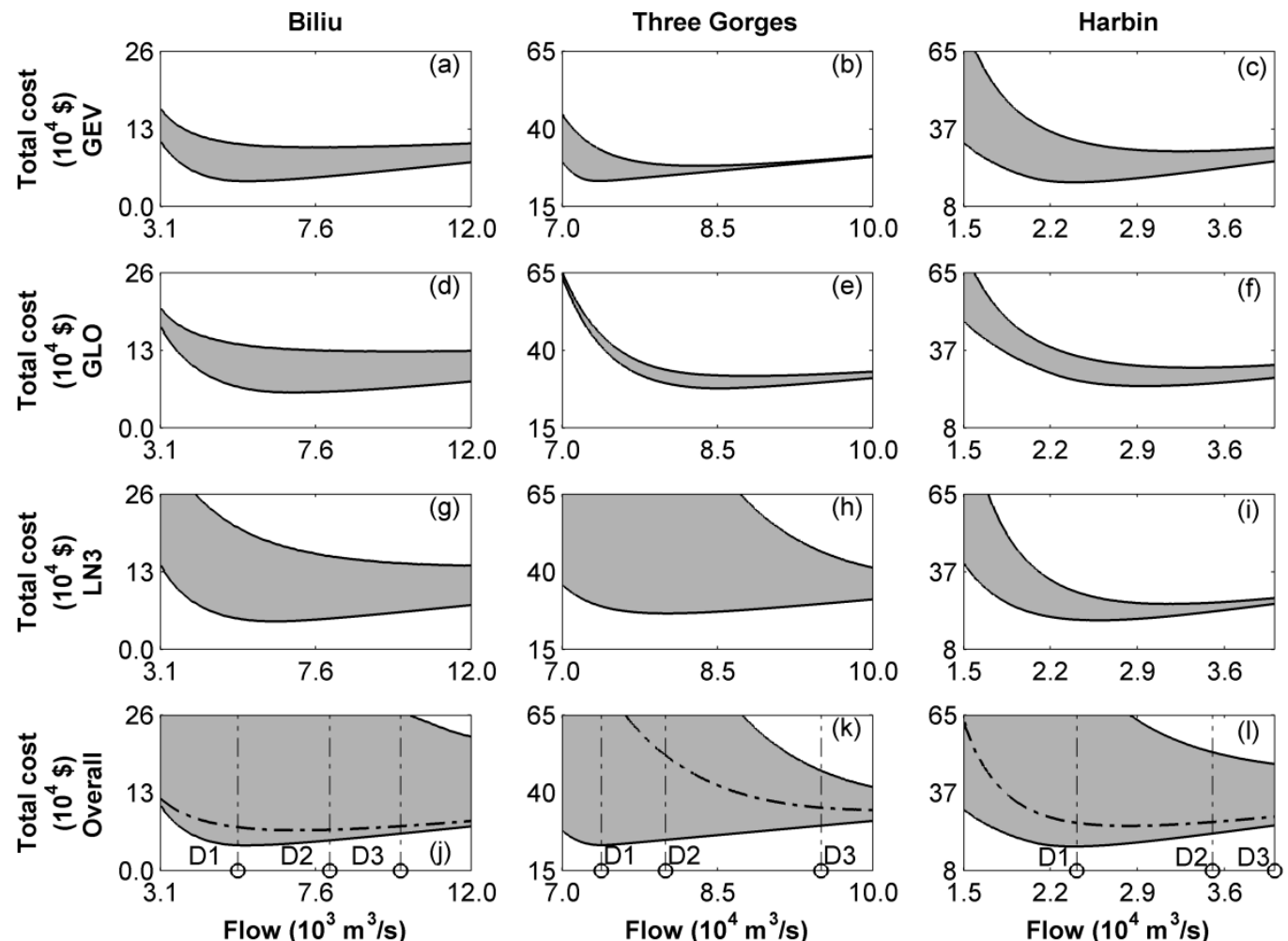

1058 Fig. 5 Lower and upper total cost bounds for 500-year, 1000-year and 500-year design flood in

1059 Biliu (a, d, g and j), Three Gorges (b, e, h and k) and Harbin (c, f, i and l) respectively. D1, D2

1060 and D3 represent three design floods. For each individual probability distribution (GEV, GLO

1061 and LN3), the uncertainty in total cost results from data selection uncertainty and probability

1062 distribution parameter uncertainty; the overall uncertainty in total cost results from selected

1063 data sets, probability distributions (GEV, GLO and LN3) and calculated parameter uncertainty. 

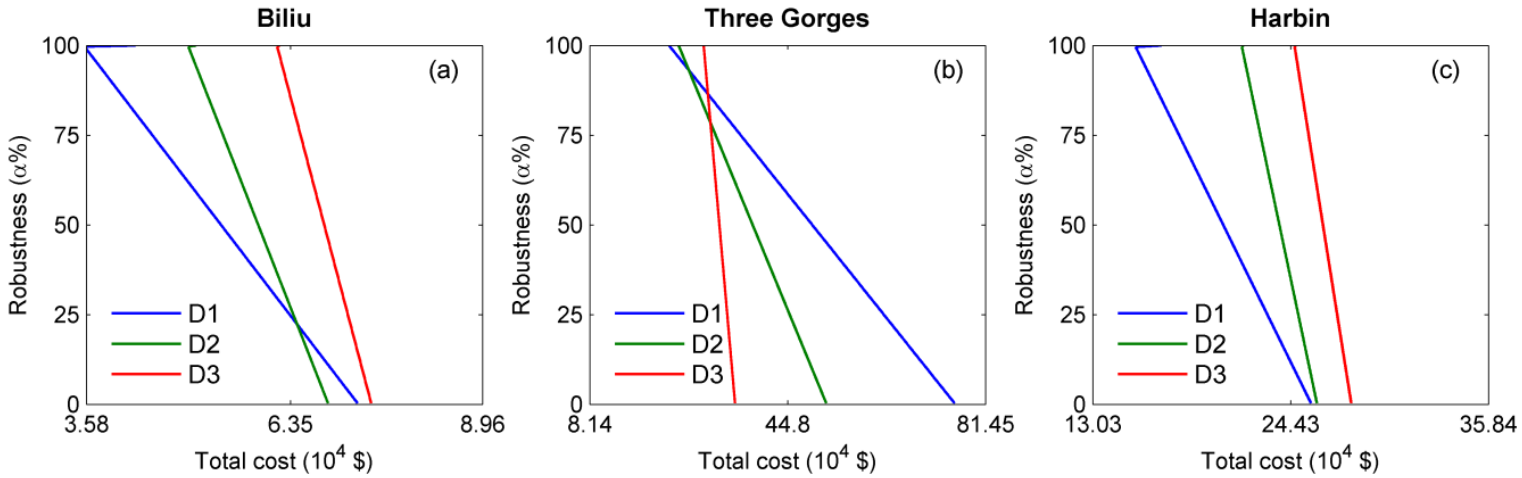

Fig. 6 Robustness of design floods under different uncertainty levels in the three case studies:

1067 Biliu (a), Three Gorges (b) and Harbin (c). Each curve represents a design flood, and its slope

1068 describes the variation of minimum total cost with uncertainty. D1, D2 and D3 represent three 1069 design flood values.

1070 

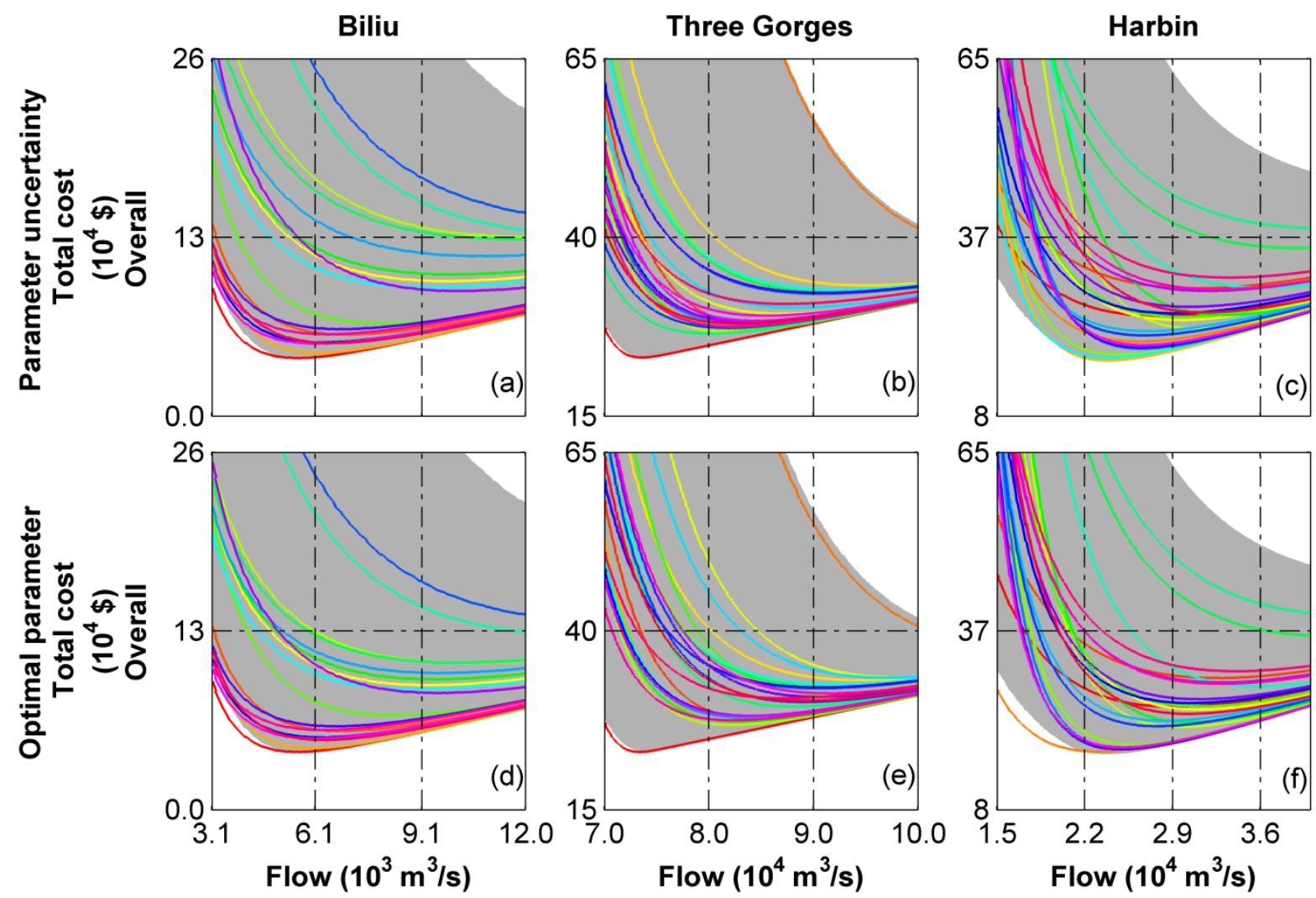

1071

1072 Fig. 7 Total cost curves of different data and probability distribution combinations (the solid

1073 lines) under two cases: with parameter uncertainty using predictive probability distributions (a,

$1074 \mathrm{~b}$ and c) and without parameter uncertainty using the estimated optimal parameters (d, e and f);

1075 total cost uncertainty bounds (the grey areas) resulting from data selection uncertainty,

1076 probability distribution uncertainty and distribution parameter uncertainty. 

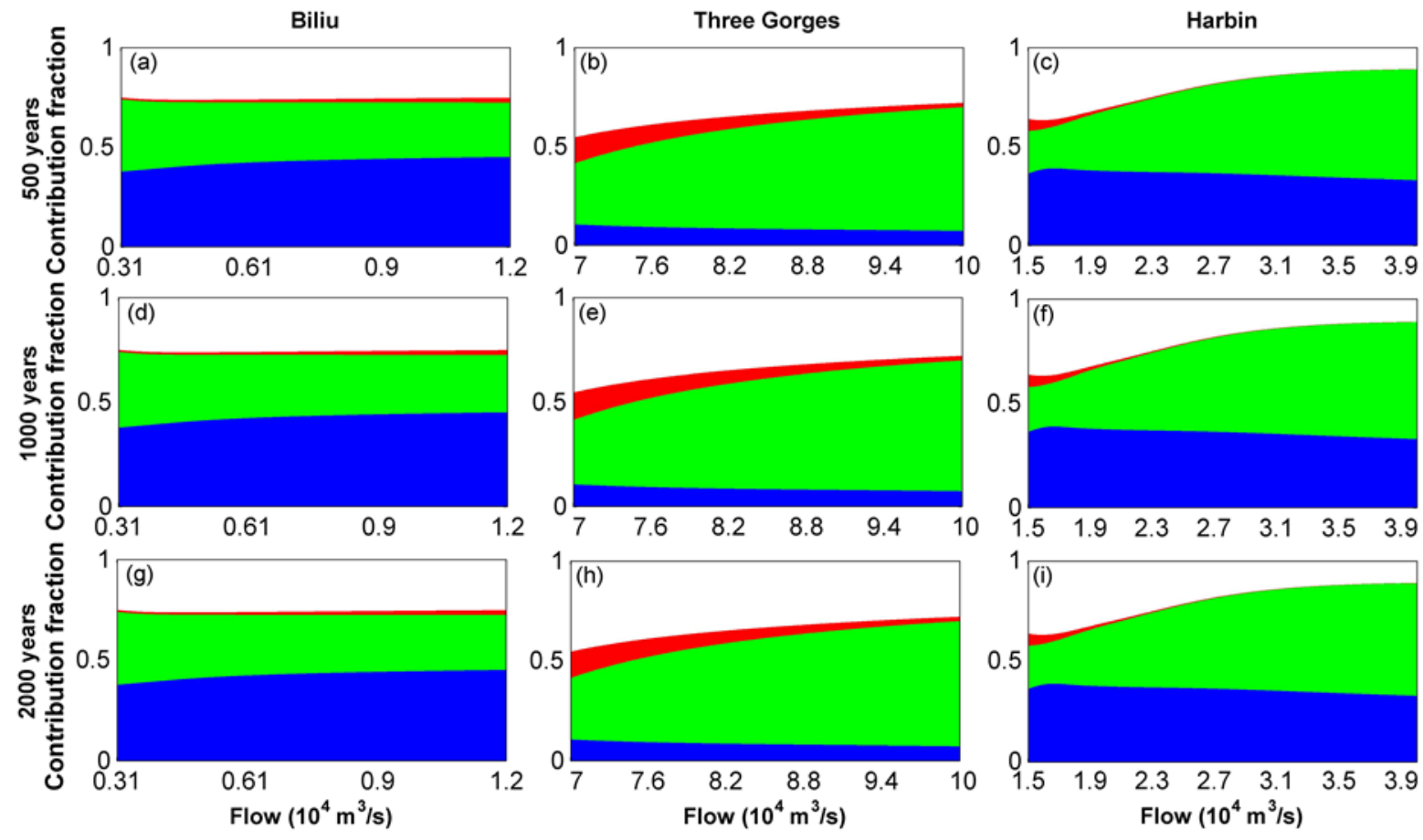

1078

Data

Distribution

Parameter Interaction

1079 Fig. 8 Contributions of uncertainty sources to the total costs of three return periods: 500, 1000

1080 and 2000 years in Biliu (a, d and g), Three Gorges (b, e and h) and Harbin (c, $\mathrm{f}$ and $\mathrm{i}$ ). The

1081 contributions of uncertainty sources are represented by the widths of the relevant strips varying

1082 with flood values on $x$-axis. 